\title{
Refractive Index as a Function of Wavelength for Sixty API-NBS Hydrocarbons
}

\author{
By Alphonse F. Forziati ${ }^{2}$
}

\begin{abstract}
Measurements of the refractive index at $20^{\circ}, 25^{\circ}$, and $30^{\circ} \mathrm{C}$ and at seven wavelengths, from 6678.1 to 4358.3 Angstrom units, were made on 60 purified hydrocarbons of the APINBS series. The apparatus consisted of a precision Abbe-type refractometer, calibrated by means of NBS Standard Samples of hydrocarbons certified with respect to refractive index at the three temperatures and seven wavelengths used. The light sources used were a mercury arc, a sodium vapor lamp, and hydrogen and helium discharge tubes, with suitable filters. A constant temperature bath maintained the temperature of the refractometer prisms constant within $\pm 0.02^{\circ} \mathrm{C}$

The experimental data on the hydrocarbons were adjusted by means of a four constant Hartmann equation, $n_{\lambda}=n_{\infty}+\mathrm{C} /\left(\lambda-\lambda^{*}\right)^{1.6}$, and a two constant modified Cauchy equation, $\Delta n_{\lambda}=a+b / \lambda^{2} . \quad\left(\Delta n_{\lambda}\right.$, the change in the refractive index at the wavelength, $\lambda$, when the temperature is changed from $20^{\circ}$ to $25^{\circ} \mathrm{C}$ or $25^{\circ}$ to $30^{\circ} \mathrm{C}$, is used instead of $n_{\lambda}$ of the Cauchy equation).

The computed refractive index at $20^{\circ}, 25^{\circ}$, and $30^{\circ} \mathrm{C}$, together with the values of the specific dispersions, and the constants of the Hartmann and modified Cauchy equations, applicable over the range of measurement, are reported for 60 API-NBS hydrocarbons, comprising 17 paraffins, 14 alkylcyclopentanes, 8 alkylcyclohexanes, and 21 alkylbenzenes.
\end{abstract}

\section{Introduction}

This investigation is part of the systematic program on the physical properties of highly purified hydrocarbons being prepared in the cooperative program of the American Petroleum Institute and the National Bureau of Standards.

Precision measurements of the refractive index were made on 60 hydrocarbons of the API-NBS series at $20^{\circ}, 25^{\circ}$, and $30^{\circ} \mathrm{C}$ and at the seven wavelengths, helium 6678.1 and $5015.7 \mathrm{~A}$, hydrogen 6562.8 and $4861.3 \mathrm{~A}$, sodium (intensityweighted mean) $5892.6 \mathrm{~A}$, and mercury 5460.7 and $4358.3 \mathrm{~A}$.

This paper gives the results of the adjustment of the data by means of the Hartmann and the modified Cauchy equations, together with the calculated specific dispersions, for 17 paraffin, 14 alkylcyclopentane, 8 alkylcyclohexane, and 21 alkylbenzene hydrocarbons.

\footnotetext{
1 This investigation was performed at the National Bureau of Standards as part of the work of the American Petroleum Institute Research Project 6 on the "Analysis, Purification, and Properties of Hydrocarbons".

2 Research Associate on the American Petroleum Institute Research Project 6 at the National Bureau of Standards.
}

\section{Apparatus and Procedure}

A precision Abbe-type Bausch and Lomb refractometer was used to measure the refractive index. In order to maintain the temperature of the refracting prism constant within the required limit of $\pm 0.02^{\circ} \mathrm{C}$, it was found necessary to remove the thermometer furnished with the instrument and to adapt a small cylinder to the water exit-tube. The top of this cylinder was threaded to accommodate a short platinum resistance thermometer (25 ohms) made specially for this purpose. $^{3}$ This cylinder permitted the water at constant temperature to flow freely through the exit-tube and provided for complete immersion of the thermometer in the water. ${ }^{4}$

A constant-temperature bath was assembled and connected to the refractometer, as shown in figure 1. The temperature of the water as it left the prism cell was measured by means of the

\footnotetext{
3 Manufactured by Cyril H. Meyers, Arlington, Va.

4 The bulb of the mercury-in-glass thermometer originally in the water exit-tube of the instrument throttled the fiow of water to such an extent that it was impossible to attain adequate temperature control.
} 
platinum resistance thermometer and a Muellertype resistance bridge. The maximum drift observed over a period of $6 \mathrm{hr}$ was $\pm 0.02 \mathrm{deg} \mathrm{C}$, whereas the temperature of the water in the bath itself varied only $\pm 0.005 \mathrm{deg}$ C. To obtain satisfactory control of temperature, it is very important that the connections from the constanttemperature bath to the refractometer be well insulated and as short as possible. Furthermore, the temperature of the water leaving the prism assembly should be measured before and after each group of measurements on each hydrocarbon and a suitable correction applied if necessary.

A diagram of the controls for the constanttemperature bath is shown in figure 2, with details given in the legend.

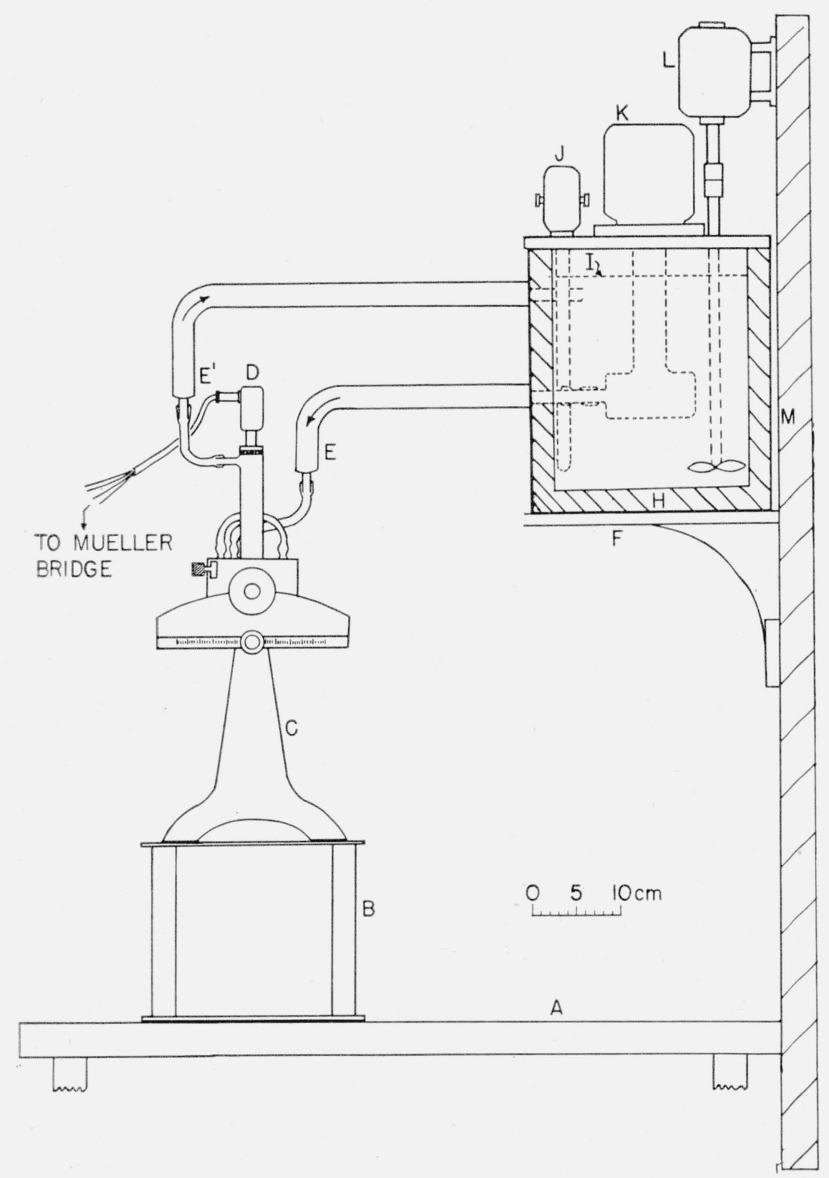

Figure 1. Assembly of precision refractometer

The letters have the following significance: A, table; B, metal supportingstand for refractometer; C, refractometer (Bausch and Lomb Precision): $D$, platinum resistance thermometer; $\mathrm{E}$, water inlet to prism assembly; $\mathrm{E}^{\prime}$, water outlet from prism assembly; F, support for constant-temperature water bath; H, thermal insulation on water-bath; I, water level; J, mercury-type thermoregulator; $\mathrm{K}$, water pump; $\mathrm{L}$, motor with stirrer; $\mathrm{M}$, wall of room.

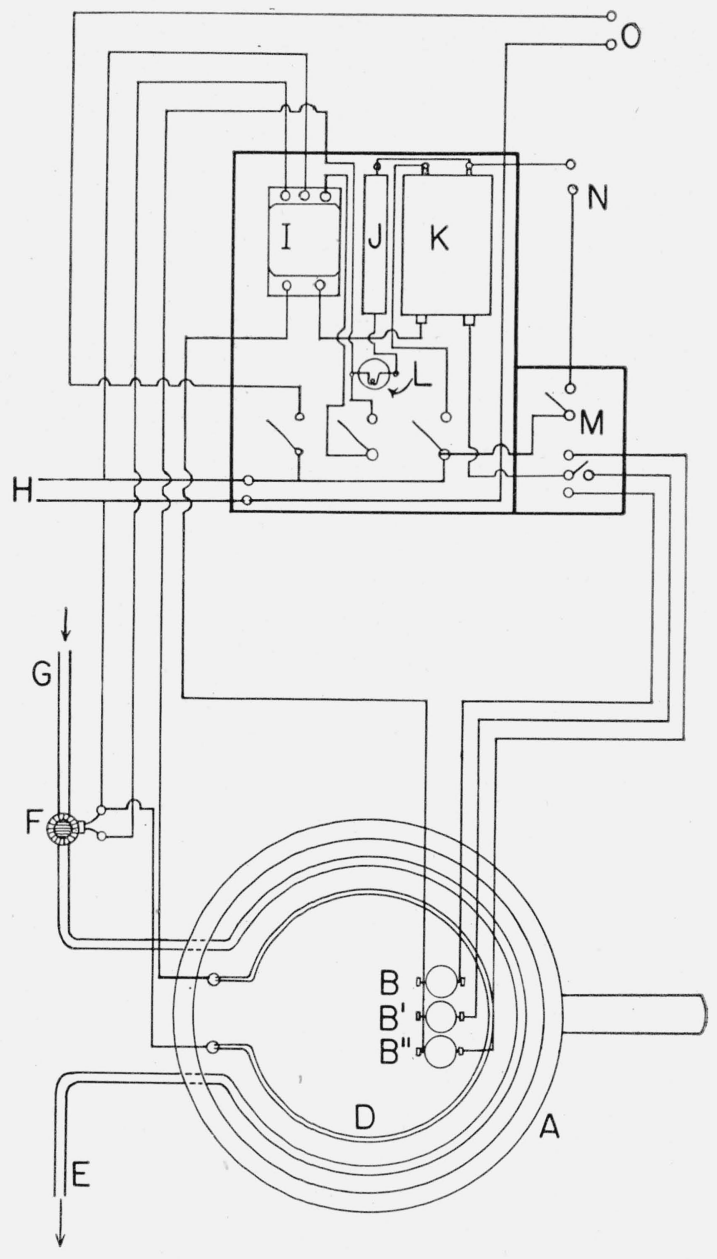

Figure 2. Diagram of controls for precision refractometer

The letters have the following significance: A, water bath; B, B', and B", mercury-type thermoregulators set at $20^{\circ} \mathrm{C}\left( \pm 0.01^{\circ} \mathrm{C}\right), 25^{\circ} \mathrm{C}\left( \pm 0.01^{\circ} \mathrm{C}\right)$, and $30^{\circ} \mathrm{C}\left( \pm 0.01^{\circ} \mathrm{C}\right)$, respectively; D, heater; $\mathrm{E}$, water discharge; $\mathrm{F}$, solenoid valve; $G$, inlet for cooling water; $H$, power line, 110 volts, alternating current; $\mathrm{I}$, relay; J, resistance, $1,000 \mathrm{ohms} ; \mathrm{K}$, rectifier and transformer; $\mathrm{L}$, miniature lamp, 14 volts; $\mathrm{M}$, switch assembly; $\mathrm{N}$, to stirrer motor; $\mathrm{O}$, to pump motor.

The sources of light used were as follows:

For the mercury lines, a mercury arc (General Electric type H4) was used. For isolating the line at $4358.3 \mathrm{~A}$, a combination of Corning filter No. 5850 (melt 647, thickness of $3.98 \mathrm{~mm}$ ) and Corning filter No. 3389 (melt 622, thickness of $3.05 \mathrm{~mm}$ ) was used. For the line at $5460.7 \mathrm{~A}$, a Wratten filter No. 62 was used.

For the hydrogen lines, a low-pressure discharge tube of the Texas Co. design [1] ${ }^{5}$ filled with hydrogen was used. A Wratten filter No. 45 was used for the line at $4861.3 \mathrm{~A}$. A Corning filter No.

\footnotetext{
${ }^{5}$ Figures in brackets indicate the literature references at the end of this paper.
} 
2404 (thickness of $3.35 \mathrm{~mm}$ ) was used for the line at $6562.8 \mathrm{~A}$ in order to improve the contrast of the light and dark portions of the field.

For the helium lines, a low-pressure discharge tube of the same type as for hydrogen, but filled with helium, was used. A Wratten filter No. 45 was used for the line at $5015.7 \mathrm{~A}$. For the line at $6678.1 \mathrm{~A}$, the same filter was used as for the hydrogen line at $6562.8 \mathrm{~A}$.

For the sodium line, intensity-weighted mean at $5892.6 \mathrm{~A}$, the standard sodium vapor lamp supplied with the refractometer was used.

The mercury arc and sodium vapor lamp were equipped with shields bearing a vertical slit, 1x25 $\mathrm{mm}$ in size.

At the beginning of each series of measurements, the refractometer was calibrated by means of the
NBS Standard Samples of 2,2,4-trimethylpentane, methylcyclohexane, and toluene, each of which is certified with respect to refractive index at $20^{\circ}$, $25^{\circ}$, and $30^{\circ} \mathrm{C}$, at each of the seven wavelengths given above. During the series of measurements, the refractometer was periodically checked with the standard used on that day. The complete calibration was repeated after a prolonged series and the change, if any, was prorated over the time interval. A table of corrections for each wavelength at $20^{\circ}, 25^{\circ}$, and $30^{\circ} \mathrm{C}$ was prepared for each one of the three standards. These corrections were then applied to the observations in accordance with the standard liquid used. The calibrating values of the three standards used, as obtained by the NBS Optical Instruments Section, are given in table 1.

TABLE 1. Certified values of refractive index for three NBS Standard Samples of Hydrocarbons

\begin{tabular}{|c|c|c|c|c|c|c|c|c|c|c|}
\hline \multirow{3}{*}{$\begin{array}{l}\text { Wavelength } \\
\text { in Angstrom } \\
\text { units }\end{array}$} & \multirow{3}{*}{ Designation of line } & \multicolumn{9}{|c|}{ Index of refraction a } \\
\hline & & \multicolumn{3}{|c|}{$\begin{array}{l}2,2,1 \text {-Trimethylpentane } \\
\text { No. } 21 i\end{array}$} & \multicolumn{3}{|c|}{$\begin{array}{c}\text { Methyleyclohexane } \\
\text { No. } 218\end{array}$} & \multicolumn{3}{|c|}{$\begin{array}{l}\text { Tolnene } \\
\text { No. 211a }\end{array}$} \\
\hline & & $20^{\circ} \mathrm{C}$ & $25^{\circ} \mathrm{C}$ & $30^{\circ} \mathrm{C}$ & $20^{\circ} \mathrm{C}$ & $25^{\circ} \mathrm{C}$ & $30 \mathrm{C}$ & $20^{\circ} \mathrm{C}$ & $25^{\circ} \mathrm{C}$ & $30^{\circ} \mathrm{C}$ \\
\hline 6678.1 & Helium ...... & 1.38916 & 1. 38670 & 1.38424 & 1. 42064 & 1. 41812 & 1.41560 & 1.49180 & 1.48903 & 1. 48619 \\
\hline 6562.8 & Hydrogen, $\mathrm{C}_{-}$ & 1.38945 & 1. 38698 & 1. 38452 & 1. 42094 & 1. 41842 & 1. 41591 & 1. 49243 & 1. 48966 & 1. 48682 \\
\hline $5892.6^{\mathrm{b}}$ & Sodium, $\mathrm{D}_{1}, \mathrm{D}_{2} \ldots$ & 1.39145 & 1. 38898 & 1. 38650 & 1. 42312 & 1. 42058 & 1. 41806 & 1. 49693 & 1. 49413 & 1. 49126 \\
\hline $5460.7 \ldots$ & Mercury, e & 1. 39316 & 1. 39068 & 1.38820 & 1. 42497 & 1. 42243 & 1. 41989 & 1. 50086 & 1.49803 & 1. 49514 \\
\hline $5015.7 \ldots$ & Helium & 1,39544 & 1. 39294 & 1. 39044 & 1. 42744 & 1. 42488 & 1. 42233 & 1. 50620 & 1. 50334 & 1,50041 \\
\hline $4861.3 \ldots$ & Hydrogen, $\mathrm{F}_{\ldots} \ldots$ & 1,39639 & 1. 39389 & 1.39138 & 1. 42847 & 1. 42590 & 1. 42334 & 1. 50847 & 1. 50559 & 1. 50265 \\
\hline $4358.3 \ldots$ & Mercury, g & 1. 40029 & 1. 39776 & 1.39523 & 1. 43269 & 1. 43010 & 1. 42752 & 1. 51800 & 1.51506 & 1. 51206 \\
\hline
\end{tabular}

a These measurements were made by the NBS Section on Optical Instruments.

b Intensity-weighted mean of doublet, $\mathrm{D}_{1}, \mathrm{D}_{2}$.

\section{Source and Purity of Compounds}

The compounds whose refractive indices were measured in the present investigation were samples from the API-NBS series of highly purified hydrocarbons, which are being prepared through a cooperative undertaking of the American Petroleum Institute and the National Bureau of Standards. The description of the compounds is given in a preceding report [2]. The purification and determination of purity and freezing points of these compounds are described in references [3, 4, 5, 6, 7]. It is believed that in each case the impurity was of such nature and present in such small amount that the properties measured were not affected beyond the indicated limits of uncertainty.

\section{Adjustment of the Data}

\section{Method of Adjustment}

The Hartmann dispersion formula, in its simple form,

$$
n_{\lambda}=n_{\infty}+C /\left(\lambda-\lambda^{*}\right),
$$

where $n_{\lambda}$ is the refractive index of the substance for light of wavelength $\lambda$, and $n_{\infty}, C$, and $\lambda^{*}$ are constants, is not sufficiently accurate for the representation of refractive indices to five decimal places. It is well known that some exponent other than unity is desirable for $\left(\lambda-\lambda^{*}\right)$ in accurate work over an extended range in wavelengths. Tilton and Gurewitz [8] found that the refractive indices of three standard hydrocarbons 
could be accurately represented to within a few units of the sixth decimal when 1.6 was used as the exponent.

The data obtained in the present investigation have been adjusted by means of the Hartmann equation as used by Tilton and Gurewitz:

$$
n_{\lambda}=n_{\infty}+C /\left(\lambda-\lambda^{*}\right)^{1.6} .
$$

The results show that the equation is entirely adequate to represent the refractive indices of a wide variety of liquid hydrocarbons over the range of wavelength from 0.66781 to $0.43583 \mu$ (or 6678 to $4358 \mathrm{~A}$ ).

The constants in eq 2 were adjusted by means of a modification ${ }^{6}$ of an interpolation precedure recommended by Tilton and Gurewitz [8]. The following abbreviations are used:

$$
\begin{gathered}
\beta=1 /\left(\lambda-\lambda^{*}\right)^{1.6}, \\
\gamma=\left(\beta_{0.43583}-\beta_{0.65628}\right) /\left(\beta_{0.43583}-\beta_{0.54607}\right) .
\end{gathered}
$$

To facilitate the calculations, values of $\beta$ and $\gamma$ were calculated over certain ranges. Table 2 gives the values of $\beta$ for each of the seven wavelengths, for values of $\lambda^{*}$ from 0.0700 to $0.1500 \mu$. Table 3 gives values of $\gamma$ for values of $\lambda^{*}$ from 0.07 to 0.15 .

\begin{tabular}{|c|c|c|c|c|c|c|c|}
\hline \multirow{3}{*}{$\lambda^{*}$} & \multicolumn{7}{|c|}{ Wavelength, $\lambda$ in microns } \\
\hline & 0.6678149 & 0. 6562793 & 0.5892620 & 0.5460740 & 0.5015675 & 0. 4861327 & 0.4358342 \\
\hline & \multicolumn{7}{|c|}{$\beta=1 /\left(\lambda-\lambda^{*}\right)^{1.6}$} \\
\hline \multirow{3}{*}{0.070} & $\beta_{\mathrm{He}-\mathrm{red}}$ & $\beta_{\mathrm{C}}$ & $\beta_{\mathrm{D}_{1}, \mathrm{D}_{2}}$ & $\beta_{\mathrm{e}}$ & $\beta_{\mathrm{He}-\mathrm{bl} \text { ue }}$ & $\beta_{\mathrm{F}}$ & $\beta_{\mathrm{g}}$ \\
\hline & 2. 277683 & 2. 349809 & 2. 85352 & 3. 278846 & 3. 836384 & 4. $06657 \mathrm{~s}$ & 4. 997411 \\
\hline & 6109 & 6427 & 881 & 11050 & 14266 & 15684 & 21934 \\
\hline \multirow[t]{2}{*}{.071} & 2. 283792 & 2. 356236 & 2.86233 & 3. 289896 & 3.850650 & 4.082262 & 5.019345 \\
\hline & 6136 & 6456 & 886 & 11110 & 14353 & 15782 & 22092 \\
\hline \multirow[t]{2}{*}{072} & 2. 289928 & 2.362692 & 2. 87119 & 3.301006 & 3.865003 & 4.098044 & 5. 041437 \\
\hline & 6163 & 6485 & 891 & 11171 & 14439 & 15882 & 22249 \\
\hline \multirow[t]{2}{*}{.073} & 2. 296091 & 2. 369177 & 2. 88010 & 3. 312177 & 3.879442 & 4.113926 & 5. 063686 \\
\hline & 6190 & 6513 & 895 & 11233 & 14528 & 15982 & 22410 \\
\hline \multirow[t]{2}{*}{.074} & 2. 302281 & 2. 375690 & 2. 88905 & 3.323410 & 3.893970 & 4. 129908 & 5. 086096 \\
\hline & 6217 & 6543 & 899 & 11295 & 14616 & 16083 & 22572 \\
\hline \multirow[t]{2}{*}{.075} & 2. 308498 & 2.382233 & 2. 89804 & 3.334705 & 3.908586 & 4.145991 & 5. 108668 \\
\hline & 6244 & 6572 & 904 & 11358 & 14705 & 16187 & 22734 \\
\hline \multirow[t]{2}{*}{.076} & 2. 314742 & 2. 388805 & 2. 90708 & 3.346063 & 3. 923291 & 4. 162178 & 5. 131402 \\
\hline & 6272 & 6601 & 909 & 11420 & 14795 & 16290 & 22900 \\
\hline \multirow[t]{2}{*}{.077} & 2. 321014 & 2. 395406 & 2. 91617 & 3.357483 & 3. 938086 & 4.178468 & 5. 154302 \\
\hline & . 6299 & 6631 & 913 & 11484 & 14887 & 16393 & 23066 \\
\hline
\end{tabular}

TABLE 2. Values of $\beta=1 /(\lambda-\lambda *)^{1.6}$, for each of seven wavelengths, $\lambda$, for the range $\lambda *=0.0700$ to 0.1500

${ }^{6}$ Harold Hurwitz, Scientific Aid on the API Research Project 6 at this Bureau, developed this modification and computed tables 2 and 3 .

${ }^{7}$ Any three wavelengths may be used to define $\gamma$. Table 3 must then be recomputed for the selected wavelengths.
TABLE 2. Values of $\beta=1 /(\lambda-\lambda *)^{1,6}$, for each of seven wavelengths, $\lambda$, for the range $\lambda^{*}=0.700$ to 0.1500 -Con.

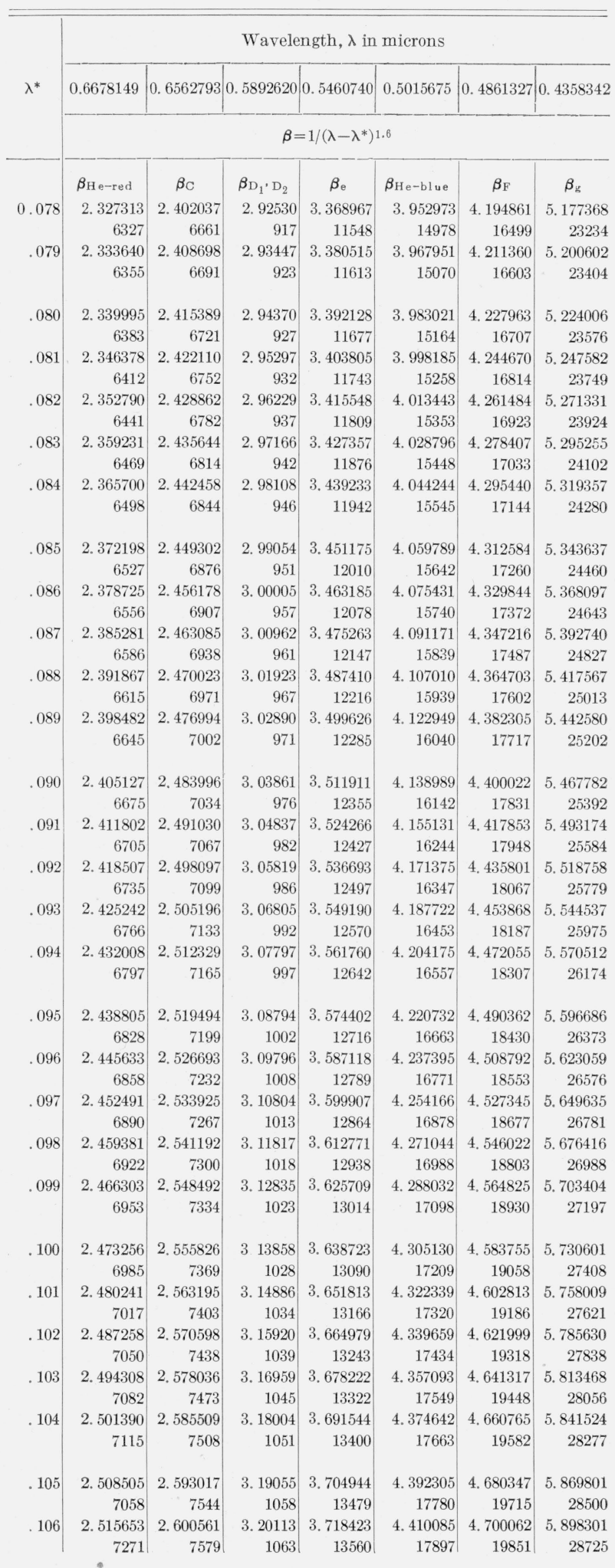


TABLE 2. Values of $\beta=1 /\left(\lambda-\lambda^{*}\right)^{1.6}$, for each of seven wavelengths, $\lambda$, for the range $\lambda^{*}=0.700$ to 0.1500 - Con.

\begin{tabular}{|c|c|c|c|c|c|c|c|}
\hline \multirow{3}{*}{$\lambda^{*}$} & \multicolumn{7}{|c|}{ Wavelength, $\lambda$ in microns } \\
\hline & 0.6678149 & 0.6562793 & 0.5892620 & 0.5460740 & 0.5015675 & 0.4861327 & 0.4358342 \\
\hline & \multicolumn{7}{|c|}{$\boldsymbol{\beta}=1 /\left(\lambda-\lambda^{*}\right) 1.6$} \\
\hline \multirow{3}{*}{0.107} & & $\beta_{\mathrm{C}}$ & & $\beta_{\mathrm{e}}$ & & $\beta_{\mathrm{F}}$ & $\beta_{\mathrm{g}}$ \\
\hline & 522834 & 2.608140 & 21176 & 3. 731983 & 4.427982 & 4. 719913 & 5. 927026 \\
\hline & 7214 & 7615 & 1070 & 13640 & 18016 & 19987 & 28953 \\
\hline \multirow[t]{2}{*}{.108} & 2. 530048 & 2.615755 & 3. 22246 & 3. 745623 & 4. 445998 & 4. 739900 & 5. 955979 \\
\hline & 7248 & 7652 & 1075 & & 18135 & 20125 & 29185 \\
\hline \multirow[t]{2}{*}{.109} & 2. 537296 & 2.623407 & 3. 23321 & 3. 759344 & 4. 464133 & 4. 760025 & 5. 985164 \\
\hline & 7282 & 7688 & 1081 & 13803 & 18255 & 20264 & 29417 \\
\hline \multirow[t]{2}{*}{.110} & 544578 & 2. 631095 & 3. 24402 & 3. 773147 & 4. 482388 & 4. 780289 & 6.014581 \\
\hline & 7316 & 7725 & 1086 & & 18376 & 20405 & 29652 \\
\hline \multirow{2}{*}{.111} & 551894 & 2. 638820 & 3. 25488 & 3. 787032 & 4. 500764 & 4. 800694 & 6.044233 \\
\hline & & & 1092 & & 18499 & 20546 & \\
\hline \multirow[t]{2}{*}{.112} & 2. 559244 & 2.646581 & 3. 26580 & 3. 8011 & 4. 519263 & 4. 821240 & 6. 074124 \\
\hline & & 7799 & 1098 & 140 & 18624 & 20690 & 30131 \\
\hline \multirow{2}{*}{.113} & 2. 566629 & 2. 654380 & 3. 27678 & 3. 815053 & 4. 537887 & 4.841930 & 6. 104255 \\
\hline & 7419 & 7836 & 1103 & 14137 & 18748 & 20835 & 30376 \\
\hline \multirow{2}{*}{.114} & 574048 & 2. 662216 & 3. 28781 & 3.829190 & 4. 556635 & 4.862765 & 6. 1346 \\
\hline & & & & & & & \\
\hline \multirow[t]{2}{*}{.115} & 2. 581 & 2.670 & 3. 29891 & 3. 8434 & 4. 575509 & 4. 883746 & 6.165253 \\
\hline & & & & & 19002 & 21129 & 871 \\
\hline \multirow[t]{2}{*}{.116} & 2. 588991 & 2.6780 & 3. 31007 & 3. 857 & 4. 594511 & 4. 904875 & 6. 196124 \\
\hline & & & & & & 21277 & 31122 \\
\hline \multirow[t]{2}{*}{.117} & 2. 596516 & 2. 685951 & 3. 32129 & 3. 872117 & 4. 613641 & 4. 926152 & 6. 227246 \\
\hline & & & & & 261 & & \\
\hline .118 & 2. 604076 & 2. 693939 & 3. 33258 & 3. 886600 & 4. 632902 & 4. 947580 & 6. 258624 \\
\hline & 7596 & 8027 & 1134 & 14572 & 19391 & 21580 & 31636 \\
\hline .119 & & 2. 701966 & 34392 & & 4. 652293 & 4.969160 & \\
\hline & & & & & 19524 & & 31897 \\
\hline .120 & 2. 619304 & 2. 710032 & 3. 35533 & 3. 9158 & 4. 671817 & 4. 990894 & 6. 322157 \\
\hline & & & & & 19657 & & \\
\hline 121 & 2. 626972 & 2. 718137 & 3. 36680 & 3. 9305 & 4. 691474 & 5. 012782 & 6. 354317 \\
\hline & & & 1154 & & 19792 & 22044 & 32426 \\
\hline .122 & 634677 & 726281 & 3. 37834 & 3.945 & 1266 & 5. 034826 & 6. 386743 \\
\hline & & & 1160 & & 19928 & 22203 & 32697 \\
\hline .123 & 642419 & 2. 734465 & 3. 38994 & 3.960354 & 4. 731194 & 5. 057029 & 6. 419440 \\
\hline & 7779 & 8225 & 1166 & 15024 & 20065 & 22362 & 32969 \\
\hline .124 & 650198 & 2. 742690 & 3. 40160 & 3. 975378 & 4. 751259 & 5. 079391 & 6. 452409 \\
\hline & & & 1173 & & 20204 & 22523 & 33246 \\
\hline .125 & 658014 & 2. 750954 & 3. 41333 & 3.990 & 4. 771463 & 5. 101914 & 6. 485655 \\
\hline & & & & & 344 & 22685 & \\
\hline .126 & 665867 & 759259 & 3. 42513 & 4. 005703 & 4. 791807 & 5.124599 & 6. 519179 \\
\hline & & & & & 20484 & 22850 & \\
\hline .127 & 673758 & 2. 767605 & 3. 43699 & 4.021007 & 4. 812291 & 5. 147449 & 6. 552986 \\
\hline & & & & & & & 34093 \\
\hline .128 & 2. 681688 & 2. 775992 & 3. 44892 & 4. 036406 & 4. 832919 & 5. 170465 & 6. 587079 \\
\hline & 7967 & 8428 & 1200 & 15496 & 20772 & 23184 & 34382 \\
\hline .129 & 689655 & 2. 784420 & 3. 46092 & 4. 051902 & 4. 853691 & 5. 193649 & \\
\hline & & & & & & & \\
\hline .130 & 2. 697661 & 2. 792890 & 3. 47299 & 4. 067494 & 4. 874608 & 5. 217002 & 6. 656136 \\
\hline & & & & & 21064 & 23524 & 34971 \\
\hline .131 & 2. 705706 & 2. 801402 & 3. 48512 & 4. 083184 & 4. 895672 & 5. 240526 & 6. 691107 \\
\hline & & & & & 21212 & 23698 & 35270 \\
\hline 132 & 2. 713790 & 2. 809956 & 3. 49732 & 4. 098974 & 4. 916884 & 264224 & 6. 726377 \\
\hline & & & 1228 & 158 & 21362 & 23872 & 35574 \\
\hline .133 & 2. 721914 & 2. 818553 & 3. 50960 & 4.114862 & 4. 938246 & 5. 288096 & 6.761951 \\
\hline & & 8640 & 1234 & 15990 & 21513 & 24049 & 35881 \\
\hline .134 & 730077 & 2. 827193 & 3. 52194 & 4. 130852 & 4. 959759 & 5. 312145 & 6. 797832 \\
\hline & & & 1241 & & 21666 & & \\
\hline & 738280 & 2. 835876 & 3. 53435 & 4. 146942 & 4. 981425 & 5. 336372 & 6. 834024 \\
\hline & 8243 & 8726 & 1248 & 16192 & 21820 & 24406 & 36504 \\
\hline
\end{tabular}

TABLE 2. Values of $\beta=1 /\left(\lambda-\lambda^{*}\right)^{1.6}$, for each of seven wavelengths, $\lambda$, for the range $\lambda^{*}=0.700$ to 0.1500 -Con.

\begin{tabular}{|c|c|c|c|c|c|c|c|}
\hline \multirow{3}{*}{$\lambda^{*}$} & \multicolumn{7}{|c|}{ Wavelength, $\lambda$ in microns } \\
\hline & 0.6678149 & 0.6562793 & 0.5892620 & 0.5460740 & 0.5015675 & 0.4861327 & 0.4358342 \\
\hline & \multicolumn{7}{|c|}{$\boldsymbol{\beta}=\mathbf{1} /\left(\lambda-\lambda^{*}\right)^{1.6}$} \\
\hline \multirow{3}{*}{0.136} & $\beta_{\mathrm{He}-\mathrm{red}}$ & $\beta_{\mathrm{C}}$ & $\beta_{\mathrm{D}_{1}, \mathrm{D}_{2}}$ & $\beta_{\ominus}$ & $\beta_{\text {He-blue }}$ & $\beta_{\mathrm{F}}$ & $\beta_{g}$ \\
\hline & 2. 746523 & 2. 844602 & 3.54683 & 4.163134 & 5.003245 & 5.360778 & 6.870528 \\
\hline & 8283 & 8770 & 1256 & 16296 & 21977 & 24588 & 36823 \\
\hline \multirow[t]{2}{*}{.137} & 2. 754806 & 2.853372 & 3. 55939 & 4. 179430 & 5. 025222 & 5. 385366 & 6.907351 \\
\hline & 8324 & 8814 & 1263 & 16399 & 22133 & 24772 & 37144 \\
\hline \multirow[t]{2}{*}{.138} & 2. 763130 & 2. 862186 & 3.57202 & 4. 195829 & 5. 047355 & 5.410138 & 6.944495 \\
\hline & 8365 & 8858 & 1270 & 16504 & 22293 & 24958 & 37471 \\
\hline \multirow[t]{2}{*}{.139} & 2. 771495 & 2.871044 & 3. 58472 & 4. 212333 & 5. 069648 & 5.435096 & 6. 981966 \\
\hline & 8406 & 8903 & 1278 & 16609 & 22453 & 25145 & 37800 \\
\hline \multirow[t]{2}{*}{.140} & 2. 779901 & 2. 879947 & 3.59750 & 4. 228942 & 5. 092101 & 5.460241 & 7. 019766 \\
\hline & 8448 & 8948 & 1285 & 16714 & 22614 & 25335 & 38133 \\
\hline \multirow[t]{2}{*}{.141} & 2. 788349 & 2. 888895 & 3. 61035 & 4. 245656 & 5.114715 & 5.485576 & 7. 057899 \\
\hline & 8489 & 8993 & 1292 & 16822 & 22779 & 25527 & 38470 \\
\hline \multirow[t]{2}{*}{.142} & 2. 796838 & 2. 897888 & 3. 62327 & 4. 262478 & 5. 137494 & 5.511103 & 7. 096369 \\
\hline & 8531 & 9039 & 1300 & 16931 & 22943 & 25720 & 38813 \\
\hline \multirow[t]{2}{*}{.143} & 2. 805369 & 2. 906927 & 3. 63627 & 4. 279409 & 5. 160437 & 5. 536823 & 7. 135182 \\
\hline & 8574 & 9084 & 1308 & 17040 & 23111 & 25916 & 39160 \\
\hline \multirow[t]{2}{*}{.144} & 2. 813943 & 2. 916011 & 3. 64935 & 4. 296449 & 5. 183548 & 5. 562739 & 7.174342 \\
\hline & 8617 & 9131 & 1315 & 17152 & 23279 & 26114 & 39510 \\
\hline \multirow[t]{2}{*}{.145} & 2.822560 & 2. 925142 & 3. 66250 & 4. 313601 & 5. 206827 & 5. 588853 & 7. 213852 \\
\hline & 8660 & 9177 & 1323 & 17265 & 23450 & 26313 & 39864 \\
\hline \multirow[t]{2}{*}{.146} & 2. 831220 & 2. 934319 & 3. 67573 & 4. 330866 & 5. 230277 & 5.615166 & 7. 253716 \\
\hline & 8703 & 9224 & 1331 & 17378 & 23621 & 26514 & 40223 \\
\hline \multirow[t]{2}{*}{.147} & 2. 839923 & 2. 943543 & 3. 68904 & 4. 348244 & 5. 253898 & 5.641680 & 7. 293939 \\
\hline & 8747 & 9271 & 1338 & 17493 & 23796 & 26719 & 40588 \\
\hline \multirow[t]{2}{*}{.148} & 2. 848670 & 2. 952814 & 3. 70242 & 4. 365737 & 5. 277694 & 5. 668399 & 7. 334527 \\
\hline & 8790 & 9319 & 1347 & 17606 & 23972 & 26926 & 40956 \\
\hline \multirow[t]{2}{*}{.149} & 2.857460 & 2. 962133 & 3. 71589 & 4. 383343 & 5. 301666 & 5.695325 & 7.375483 \\
\hline & 8835 & 9367 & 1354 & 17723 & 24149 & 27134 & 41329 \\
\hline .150 & 2.866295 & 2. 971500 & 3. 72943 & 4. 401066 & 5. 325815 & 5.722459 & 7. 416812 \\
\hline
\end{tabular}

The value of $\gamma$ was determined from the relation

$$
\gamma=\left(n_{0.43583}-n_{0.65628}\right) /\left(n_{0.43583}-n_{0.54607}\right) .
$$

Using table 3 , the corresponding value of $\lambda^{*}$ was interpolated. Further interpolation in table 2 yielded values for $\beta$ for each of the seven wavelengths used. The constant $C$ was then readily computed from the equation

$$
C=\left(n_{0.43583}-n_{0.65628}\right) /\left(\beta_{0.43583}-\beta_{0.65628}\right) .
$$

The Hartmann equation was written as

$$
n_{\lambda}=n_{\infty}+C \beta_{\lambda} .
$$

The remaining unknown constant, $n_{\infty}$, was easily computed by inserting the observed values of the refractive index at $\lambda=0.65628,0.54607$, and $0.43583 \mu$, the numerical value of $C$, and appropriate values of $\beta_{\lambda}$, successively into the above 
equation and solving three times for $n_{\infty}$. If no errors were made, the three computed values of $n_{\infty}$ agreed within \pm 0.00001 . The same equation was used to compute the refractive index at $\lambda=0.66781,0.58926,0.50157$, and $0.48613 \mu$, by inserting the average value of $n_{\infty}$, and the values of $C$ and $\beta_{\lambda}$. Since it was found that this pro- cedure reproduces good data to about \pm 0.00001 units in the refractive index, it is obvious that attempts to better these constants of the Hartmann equation by least squares or any other method of adjustment would be of no practical value in this work where only fifth decimal data is to be considered.

TABLE 3. Values of $\gamma=\left(\beta_{0.48698}-\beta_{0.65688}\right) /\left(\beta_{0.48583}-\beta_{0.54607}\right)$, for values of $\lambda$ * from 0.07 to 0.15

\begin{tabular}{|c|c|c|c|c|c|c|c|c|}
\hline \multirow{3}{*}{$\begin{array}{c}\text { Intervals } \\
\text { of } \lambda^{*}\end{array}$} & \multicolumn{8}{|c|}{$\lambda^{*}$} \\
\hline & 0.07000 & 0.08000 & 0.09000 & 0.10000 & 0.11000 & 0.12000 & 0.13000 & 0.14000 \\
\hline & \multicolumn{8}{|c|}{$\gamma=\left(\boldsymbol{\beta}_{0.43583}-\boldsymbol{\beta}_{0.65628}\right) /\left(\boldsymbol{\beta}_{0.43583}-\boldsymbol{\beta}_{0.54607}\right)$} \\
\hline \multirow[t]{2}{*}{0.000} & 1. 540589 & 1. 533190 & 1. 525554 & 1. 517667 & 1. 509518 & 1. 501096 & 1. 492383 & 1. 483368 \\
\hline & 729 & 753 & 778 & 802 & 830 & 858 & 888 & 920 \\
\hline \multirow[t]{2}{*}{.001} & 1. 539860 & 1. 532437 & 1. 524776 & 1. 516865 & 1. 508688 & 1. 500238 & 1. 491495 & 1. 482448 \\
\hline & 733 & 755 & 779 & 806 & 832 & 860 & 890 & 923 \\
\hline \multirow[t]{2}{*}{.002} & 1. 539127 & 1. 531682 & 1. 523997 & 1. 516059 & 1. 507856 & 1. 499378 & 1. 490605 & 1. 481525 \\
\hline & .734 & 757 & 783 & 809 & 835 & 864 & 894 & 926 \\
\hline \multirow[t]{2}{*}{.003} & 1. 538393 & 1. 530925 & 1. 523214 & 1. 515250 & 1. 507021 & 1. 498514 & 1. 489711 & 1. 480599 \\
\hline & 736 & 761 & 785 & 810 & 838 & 867 & 896 & 929 \\
\hline \multirow[t]{2}{*}{.004} & 1. 537657 & 1. 530164 & 1. 522429 & 1. 514440 & 1. 506183 & 1. 497647 & 1. 488815 & 1. 479670 \\
\hline & 739 & 762 & 787 & 814 & 841 & 869 & 901 & 932 \\
\hline \multirow[t]{2}{*}{.005} & 1. 536918 & 1. 529402 & 1. 521642 & 1. 513626 & 1. 505342 & 1. 496778 & 1. 487914 & 1. 478738 \\
\hline & 741 & 765 & 789 & 817 & 844 & 874 & 903 & 935 \\
\hline \multirow[t]{2}{*}{.006} & 1. 536177 & 1. 528637 & 1. 520853 & 1. 512809 & 1. 504498 & 1. 495904 & 1. 487011 & 1. 477803 \\
\hline & 744 & 767 & 793 & 818 & 846 & 875 & 905 & 937 \\
\hline \multirow[t]{2}{*}{.007} & 1. 535433 & 1. 527870 & 1. 520060 & 1. 511991 & 1. 503652 & 1. 495029 & 1. 486106 & 1. 476866 \\
\hline & 745 & 769 & 795 & 821 & 849 & 879 & 910 & $\begin{array}{r}1 \quad 940\end{array}$ \\
\hline \multirow[t]{2}{*}{.008} & 1. 534688 & 1. 527101 & 1. 519265 & 1. 511170 & 1. 502803 & 1. 494150 & 1. 485196 & 1. 475926 \\
\hline & 748 & 773 & 798 & 825 & 852 & 882 & 912 & 945 \\
\hline \multirow[t]{2}{*}{.009} & 1. 533940 & 1. 526328 & 1. 518467 & 1. 510345 & 1. 501951 & 1. 493268 & 1. 484284 & 1. 474981 \\
\hline & 750 & 774 & 800 & 827 & 855 & 885 & 916 & 947 \\
\hline
\end{tabular}

Instead of applying the above procedure separately to the data obtained at $20^{\circ}, 25^{\circ}$, and $30^{\circ} \mathrm{C}$, it was decided that the 21 measurements on each hydrocarbon would be better correlated if the change in refractive index in going from $20^{\circ}$ to $25^{\circ} \mathrm{C}$ and from $25^{\circ}$ to $30^{\circ} \mathrm{C}$ were first adjusted by means of the method of least squares and the Cauchy formula

$$
\Delta n_{\lambda}=a+b / \lambda^{2},
$$

using $\Delta n_{\lambda}$ instead of $n_{\lambda}$.

The computed values of $\Delta n$, for each wavelength, were then added to the $30^{\circ} \mathrm{C}$ refractive indices and subtracted from the $20^{\circ} \mathrm{C}$ indices to give two values at $25^{\circ} \mathrm{C}$. These were averaged with the experimentally determined indices of refraction at $25^{\circ} \mathrm{C}$, and the average fitted to the Hartmann equation by the interpolation method described above. By adding and subtracting the computed values of $\Delta n$ from the refractive indices calculated by means of the constants of the Hartmann equation, the values of refractive index for $20^{\circ}$ and $30^{\circ} \mathrm{C}$ were obtained. This procedure automatically "smoothed" the values for $20^{\circ}$ and $30^{\circ} \mathrm{C}$. In the case of the paraffins and cycloparaffins, the values of $\Delta n$ for the range $20^{\circ}$ to $25^{\circ} \mathrm{C}$ were so nearly equal to those for the range $25^{\circ}$ to $30^{\circ} \mathrm{C}$ that average values of $\Delta n$ were used.

\section{Results of the Adjustment}

Table 4 gives the values of the constants of the modified Cauchy equation for each of the $60 \mathrm{com}-$ pounds. The last column of the table gives the root-mean-square value, $\rho \times 10^{5}$, of the deviations of the observed from the calculated refractive indices.

Table 5 gives the values of the constants of the Hartmann equation for each of the 60 compounds. As before, the last column of the table gives the root-mean-square value, $\rho \times 10^{5}$, of the deviations 
of the observed from the calculated refractive indices.

Table 6 gives the calculated values of refractive index at $20^{\circ}, 25^{\circ}$, and $30^{\circ} \mathrm{C}$, at each of seven wavelengths, for each of the 60 compounds.
Table 7 gives values of the specific dispersion, $10^{4}\left(n_{F}-n_{C}\right) / d$ and $10^{4}\left(n_{g}-n_{D}\right) / d$, for each of the 60 compounds, from the values of refractive index given in this report and the values of density given in an earlier report [2].

TABLE 4. Values of the constants of the modified Cauchy equation for 60 API-NBS hydrocarbons

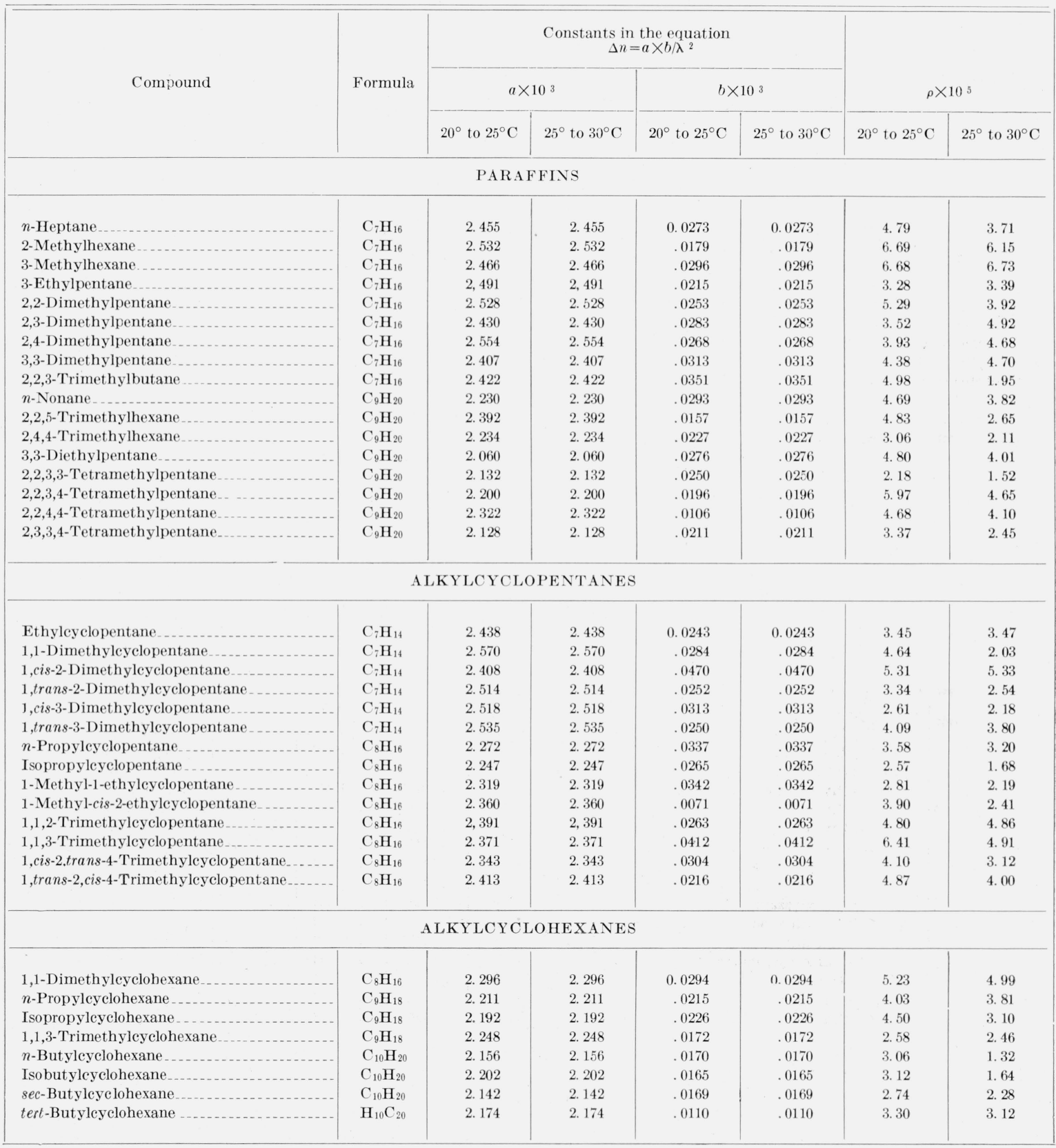


TABLE 4. Values of the constants of the modified Cauchy equation for 60 API-NBS hydrocarbons-Continued

\begin{tabular}{|c|c|c|c|c|c|c|c|}
\hline \multirow{3}{*}{ Compound } & \multirow{3}{*}{ Formula } & \multicolumn{4}{|c|}{$\begin{array}{l}\text { Constants in the equation } \\
\qquad \Delta n=a+b / \lambda^{2}\end{array}$} & & \\
\hline & & \multicolumn{2}{|c|}{$a \times 10^{3}$} & \multicolumn{2}{|c|}{$b \times 10^{3}$} & \multicolumn{2}{|c|}{$\rho \times 10^{5}$} \\
\hline & & $20^{\circ}$ to $25^{\circ} \mathrm{C}$ & $25^{\circ}$ to $30^{\circ} \mathrm{C}$ & $20^{\circ}$ to $25^{\circ} \mathrm{C}$ & $25^{\circ}$ to $30^{\circ} \mathrm{C}$ & $20^{\circ}$ to $25^{\circ} \mathrm{C}$ & $25^{\circ}$ to $30^{\circ} \mathrm{C}$ \\
\hline \multicolumn{8}{|c|}{ ALKYLBENZENES } \\
\hline Benzene.......... & $\mathrm{C}_{6} \mathrm{H}_{6}$ & 2. 988 & 3.020 & 0.0753 & 0.0424 & 0.94 & 1. 97 \\
\hline Methylbenzene & $\mathrm{C}_{7} \mathrm{H}_{8}$ & 2. 658 & 2. 704 & .0459 & .0620 & 2.89 & 1. 28 \\
\hline Ethylbenzene & $\mathrm{C}_{8} \mathrm{H}_{10}$ & 2,541 & 2. 617 & .0501 & .0290 & 7.70 & 0.80 \\
\hline 1,2-Dimethylbenzene & $\mathrm{C}_{8} \mathrm{H}_{10}$ & 2. 331 & 2. 503 & .0590 & .0432 & 1.55 & 1.49 \\
\hline 1,3-Dimethylbenzene & $\mathrm{C}_{8} \mathrm{H}_{10}$ & 2. 383 & 2.585 & .0669 & .0255 & 1.90 & 1.57 \\
\hline 1,4-Dimethylbenzene & $\mathrm{C}_{8} \mathrm{H}_{10}$ & 2. 394 & 2. 616 & .0595 & .0325 & 2. 62 & 1.02 \\
\hline$n$-Propylbenzene & $\mathrm{C}_{9} \mathrm{H}_{12}$ & 2. 372 & 2. 382 & .0494 & .0368 & 1.39 & 1.54 \\
\hline Isopropylbenzene & $\mathrm{C}_{9} \mathrm{H}_{12}$ & 2. 428 & 2. 328 & .0419 & .0398 & 3.21 & 1.34 \\
\hline 1-Methyl-2-ethylbenzene & $\mathrm{C}_{9} \mathrm{H}_{12}$ & 2. 340 & 2. 396 & .0478 & .0193 & 3.86 & 2.12 \\
\hline 1-Methyl-3-ethylbenzene... & $\mathrm{C}_{9} \mathrm{H}_{12}$ & 2. 390 & 2. 439 & .0535 & .0271 & 1.54 & 3. 22 \\
\hline 1-Methyl-4-ethylbenzene . . & $\mathrm{C}_{9} \mathrm{H}_{12}$ & 2. 428 & 2. 348 & .0443 & .0356 & 1. 20 & 1.94 \\
\hline 1,2,3-Trimethylbenzene & $\mathrm{C}_{9} \mathrm{H}_{12}$ & 2. 320 & 2. 341 & .0467 & .0184 & 1.91 & 1.75 \\
\hline 1,2,4-Trimethylbenzene & $\mathrm{C}_{9} \mathrm{H}_{12}$ & 2. 337 & 2. 337 & .0457 & .0457 & 2.81 & 1.93 \\
\hline 1,3,5-Trimethylbenzene & $\mathrm{C}_{9} \mathrm{H}_{12}$ & 2. 445 & 2. 341 & .0303 & .0431 & 1.52 & 1.95 \\
\hline$n$-Butylbenzene & $\mathrm{C}_{10} \mathrm{H}_{14}$ & 2. 246 & 2. 376 & .0421 & .0075 & 2.78 & 2.61 \\
\hline Isobutylbenzene & $\mathrm{C}_{10} \mathrm{H}_{14}$ & 2. 347 & 2. 348 & .0407 & .0294 & 2.41 & 1.80 \\
\hline sec-Butylbenzene & $\mathrm{C}_{10} \mathrm{H}_{14}$ & 2. 280 & 2. 354 & .0443 & .0220 & 1.84 & 1.59 \\
\hline tert-Butylbenzene ..... & $\mathrm{C}_{10} \mathrm{H}_{14}$ & 2. 306 & 2. 348 & .0395 & .0443 & 3. 24 & 3.06 \\
\hline 1,2-Diethylbenzene.... & $\mathrm{C}_{10} \mathrm{H}_{14}$ & 2. 249 & 2. 370 & .0527 & .0147 & 1. 98 & 1.91 \\
\hline 1,3-Diethylbenzene & $\mathrm{C}_{10} \mathrm{H}_{14}$ & 2. 282 & 2. 334 & .0469 & .0348 & 0.91 & 2.03 \\
\hline 1,4-Diethylbenzene & $\mathrm{C}_{10} \mathrm{H}_{14}$ & 2. 223 & 2. 353 & .0537 & .0234 & 1.30 & 1.05 \\
\hline
\end{tabular}

TABLE 5. Values of the constants of the Hartmann equation for the 60 API-NBS hydrocarbons

\begin{tabular}{|c|c|c|c|c|c|}
\hline \multirow{2}{*}{ Compound } & \multirow{2}{*}{ Formula } & \multicolumn{3}{|c|}{$\begin{array}{c}\text { Constants in the equation } \\
n_{\lambda}=n_{\infty}+\boldsymbol{C} /\left(\lambda-\lambda^{*}\right)^{1.6} \text { at } 25^{\circ} \mathrm{C}\end{array}$} & \multirow[b]{2}{*}{$\rho$} \\
\hline & & $n_{\infty}$ & $C$ & $\lambda^{*}$ & \\
\hline \multicolumn{6}{|c|}{ PARAFFINS } \\
\hline$n$-Heptane $\ldots \ldots$ & $\mathrm{C}_{7} \mathrm{H}_{16}$ & 1. 37475 & 0.003341 & 0.09641 & 1. 49 \\
\hline 2-Methylhexane ....... & $\mathrm{C}_{7} \mathrm{H}_{16}$ & 1. 37195 & .003307 & .09832 & 1.34 \\
\hline 3-Methylhexane & $\mathrm{C}_{7} \mathrm{H}_{16}$ & 1. 37570 & .003348 & .09649 & 1.02 \\
\hline 3-Ethylpentane & $\mathrm{C}_{7} \mathrm{H}_{16}$ & 1. 38046 & .003357 & .09544 & 1. 15 \\
\hline 2,2-Dimethylpentane ..... & $\mathrm{C}_{7} \mathrm{H}_{16}$ & 1. 36927 & .003274 & .10022 & 0.87 \\
\hline 2,3-Dimethylpentane . . ..... & $\mathrm{C}_{7} \mathrm{H}_{16}$ & 1. 37920 & .003254 & .10122 & 1.46 \\
\hline 2,4-Dimethylpentane ..... & $\mathrm{C}_{7} \mathrm{H}_{16}$ & 1. 36860 & .003266 & .09907 & 1. 12 \\
\hline 3,3-Dimethylpentane .... & $\mathrm{C}_{7} \mathrm{H}_{16}$ & 1. 37781 & .003494 & .08991 & 1. 30 \\
\hline 2,2,3-Trimethylbutane & $\mathrm{C}_{7} \mathrm{H}_{16}$ & 1. 37620 & .003536 & .08921 & 1. 22 \\
\hline$n$-Nonane & $\mathrm{C}_{9} \mathrm{H}_{20}$ & 1. 39243 & .003381 & 10202 & 1. 68 \\
\hline 2,2,5-Trimethylhexane & $\mathrm{C}_{9} \mathrm{H}_{20}$ & 1. 38657 & .003389 & .10208 & 0.93 \\
\hline 2,4,4-Trimethylhexane & $\mathrm{C}_{9} \mathrm{H}_{20}$ & 1. 39411 & .003560 & .09624 & .37 \\
\hline 3,3-Diethylpentane & $\mathrm{C}_{9} \mathrm{H}_{20}$ & 1. 40733 & .003560 & .09624 & 1.07 \\
\hline 2,2,3,3-Tetramethylpentane & $\mathrm{C}_{9} \mathrm{H}_{20}$ & 1. 41030 & .003503 & 10282 & 0.93 \\
\hline 2,2,3,4-Tetramethylpentane & $\mathrm{C}_{9} \mathrm{H}_{20}$ & 1. 40146 & .003494 & .10082 & .84 \\
\hline 2,2,4,4-Tetramethylpentane & $\mathrm{C}_{9} \mathrm{H}_{20}$ & 1. 39343 & .003543 & .10116 & 2. 32 \\
\hline 2,3,3,4-Tetramethylpentane & $\mathrm{C}_{9} \mathrm{H}_{20}$ & 1. 40880 & .003617 & .09667 & 1. 28 \\
\hline
\end{tabular}


TABLE 5. Values of the constants of the Hartmann equation for the 60 API-NBS hydrocarbons-Continued

\begin{tabular}{|c|c|c|c|c|c|}
\hline \multirow{2}{*}{ Compound } & \multirow{2}{*}{ Formula } & \multicolumn{3}{|c|}{$\begin{array}{c}\text { Constants in the equation } \\
n_{\lambda}=n_{\infty}+C /\left(\lambda-\lambda^{*}\right)^{1.6} \text { at } 25^{\circ} \mathrm{C}\end{array}$} & \multirow[b]{2}{*}{$\rho$} \\
\hline & & $n_{\infty}$ & $C$ & $\lambda^{*}$ & \\
\hline \multicolumn{6}{|c|}{ ALKYLCYCLOPENTANES } \\
\hline Ethylcyclopentane & $\mathrm{C}_{7} \mathrm{H}_{14}$ & 1. 40579 & 0.003778 & 0.09084 & 0.62 \\
\hline 1,1-Dimethylcyclopentane ........ & $\mathrm{C}_{7} \mathrm{H}_{14}$ & 1. 39941 & .003750 & .09273 & 1.15 \\
\hline 1,cis-2-Dimethylcyclopentane $\ldots . . . . .$. & $\mathrm{C}_{7} \mathrm{H}_{14}$ & 1. 40729 & .004306 & .07150 & 3. 31 \\
\hline 1,trans-2-Dimethylcyclopentane $\ldots . .$. & $\mathrm{C}_{7} \mathrm{H}_{14}$ & 1. 39830 & .003559 & .09830 & 1. 78 \\
\hline 1,cis-3-Dimethylcyclopentane & $\mathrm{C}_{7} \mathrm{H}_{14}$ & 1. 39687 & .003669 & .09299 & 1. 10 \\
\hline 1, trans-3-Dimethylcyclopentane $\ldots . . .$. & $\mathrm{C}_{7} \mathrm{H}_{14}$ & 1. 39522 & .003559 & .09830 & 2.42 \\
\hline$n$-Propyleyclopentane $\ldots$ & $\mathrm{C}_{8} \mathrm{H}_{16}$ & 1. 41247 & .003640 & .09991 & 1. 03 \\
\hline Isopropylcyclopentane & $\mathrm{C}_{8} \mathrm{H}_{16}$ & 1. 41210 & .003624 & .10076 & 1. 22 \\
\hline 1-Methyl-1-ethylcyclopentane $\ldots . . . . .$. & $\mathrm{C}_{8} \mathrm{H}_{16}$ & 1. 41300 & .003850 & .09160 & 0.80 \\
\hline Ethylcyclopentane & $\mathrm{C}_{8} \mathrm{H}_{16}$ & 1. 41529 & .003799 & .09320 & .84 \\
\hline 1,1,2-Trimethyleyclopentane ........... & $\mathrm{C}_{8} \mathrm{H}_{16}$ & 1. 40888 & .003755 & .09589 & .54 \\
\hline 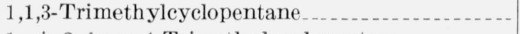 & $\mathrm{C}_{8} \mathrm{H}_{16}$ & 1. 39691 & .003956 & .08384 & 1. 45 \\
\hline 1, cis-2, trans-4-Trimethyleyclopentane .......... & $\mathrm{C}_{8} \mathrm{H}_{16}$ & 1. 40481 & .003641 & 09694 & 0.68 \\
\hline 1, trans-2, cis-4-Trimethyleyclopentane & $\mathrm{C}_{8} \mathrm{H}_{16}$ & 1. 39683 & .003668 & .09405 & 1.17 \\
\hline \multicolumn{6}{|c|}{ ALKYLCYCLOHEXANES } \\
\hline 1,1-Dimethylcyclohexane... & $\mathrm{C}_{8} \mathrm{H}_{16}$ & 1. 41434 & 0.004109 & 0.08469 & 0.87 \\
\hline n-Propylcyclohexane $\ldots . .$. & $\mathrm{C}_{9} \mathrm{H}_{18}$ & 1. 42266 & .003954 & .09262 & 1. 92 \\
\hline Isopropylcyclohexane.......... & $\mathrm{C}_{9} \mathrm{H}_{18}$ & 1. 42653 & .003919 & .09432 & 0.78 \\
\hline 1,1,3-Trimethylcyclohexane $\ldots .$. & $\mathrm{C}_{9} \mathrm{H}_{18}$ & 1. 41535 & .003803 & .09917 & .84 \\
\hline n-Butyleyclohexane $\ldots . . .$. & $\mathrm{C}_{10} \mathrm{H}_{20}$ & 1. 42646 & .003911 & .09522 & .69 \\
\hline Isobutylcyclohexane ....... & $\mathrm{C}_{10} \mathrm{H}_{20}$ & 1. 42431 & .003877 & .09689 & 1. 31 \\
\hline sec-Butylcyclohexane & $\mathrm{C}_{10} \mathrm{H}_{20}$ & 1. 43238 & .003942 & .09460 & 1. 54 \\
\hline tert-Butylcyclohexane & $\mathrm{C}_{10} \mathrm{H}_{20}$ & 1. 43248 & .003959 & .09563 & 0.89 \\
\hline \multicolumn{6}{|c|}{ ALKYLBENZENES } \\
\hline Benzene ......... & $\mathrm{C}_{6} \mathrm{H}_{6}$ & 1. 47421 & 0.006825 & 0.13003 & 0.60 \\
\hline Methylbenzene........... & $\mathrm{C}_{7} \mathrm{H}_{8}$ & 1. 47132 & .006556 & .13046 & .20 \\
\hline Ethylbenzene $\ldots . . . . . . .$. & $\mathrm{C}_{8} \mathrm{H}_{10}$ & 1. 47152 & .006276 & .12843 & 3. 08 \\
\hline 1,2-Dimethylbenzene $\ldots \ldots \ldots$ & $\mathrm{C}_{8} \mathrm{H}_{10}$ & 1. 48037 & .006500 & .13012 & 1. 48 \\
\hline 1,3-Dimethylbenzene . . . . . . . & $\mathrm{C}_{8} \mathrm{H}_{10}$ & 1. 47249 & .006349 & .13126 & 0.87 \\
\hline 1,4-Dimethylbenzene ........ & $\mathrm{C}_{8} \mathrm{H}_{10}$ & 1. 47098 & .006393 & 13089 & 1. 79 \\
\hline n-Propylbenzene $\ldots \ldots$ & $\mathrm{C}_{9} \mathrm{H}_{12}$ & 1. 46895 & .005968 & .12771 & 1.98 \\
\hline Isopropylbenzene & $\mathrm{C}_{9} \mathrm{H}_{12}$ & 1. 46850 & .005898 & .12886 & 2. 51 \\
\hline 1-Methyl-2-ethylbenzene & $\mathrm{C}_{9} \mathrm{H}_{12}$ & 1. 48026 & .006355 & .12666 & 1. 73 \\
\hline 1-Methyl-3-ethylbenzene....... & $\mathrm{C}_{9} \mathrm{H}_{12}$ & 1. 47258 & .006239 & .12745 & 1. 97 \\
\hline 1-Methyl-4-ethylbenzene & $\mathrm{C}_{9} \mathrm{H}_{12}$ & 1. 47114 & .006126 & 13028 & 1. 09 \\
\hline 1,2,3-Trimethylbenzene $\ldots \ldots \ldots$ & $\mathrm{C}_{9} \mathrm{H}_{12}$ & 1. 48915 & .006406 & 13118 & 1.07 \\
\hline 1,2,4-Trimethylbenzene $\ldots . . .$. & $\mathrm{C}_{9} \mathrm{H}_{12}$ & 1. 48024 & .006337 & .13160 & 1. 10 \\
\hline 1,3,5-Trimethylbenzene & $\mathrm{C}_{9} \mathrm{H}_{12}$ & 1. 47501 & .006249 & .13173 & 0.78 \\
\hline$n$-Butylbenzene & $\mathrm{C}_{10} \mathrm{H}_{14}$ & 1. 46761 & .005805 & .12487 & 2. 92 \\
\hline Iso butylbenzene........... & $\mathrm{C}_{10} \mathrm{H}_{14}$ & 1. 46418 & .005817 & .12448 & 1. 37 \\
\hline sec-Butylbenzene...... & $\mathrm{C}_{10} \mathrm{H}_{14}$ & 1. 46803 & .005784 & .12527 & 0.84 \\
\hline tert-Butylbenzene ...... & $\mathrm{C}_{10} \mathrm{H}_{14}$ & 1. 47032 & .005838 & 12485 & 2. 66 \\
\hline 1,2-Diethylbenzene $\ldots$ & $\mathrm{C}_{10} \mathrm{H}_{14}$ & 1. 48014 & .006059 & 12832 & 1. 18 \\
\hline 1,3-Diethylbenzene & $\mathrm{C}_{10} \mathrm{H}_{14}$ & 1. 47241 & .006013 & 12731 & 2. 55 \\
\hline 1,4-Diethylbenzene & $\mathrm{C}_{10} \mathrm{H}_{14}$ & 1. 47172 & .006001 & 12838 & 1. 71 \\
\hline
\end{tabular}


TABLE 6. Refractive indices at seven wavelengths and three temperatures for 60 API-NBS hydrocarbons

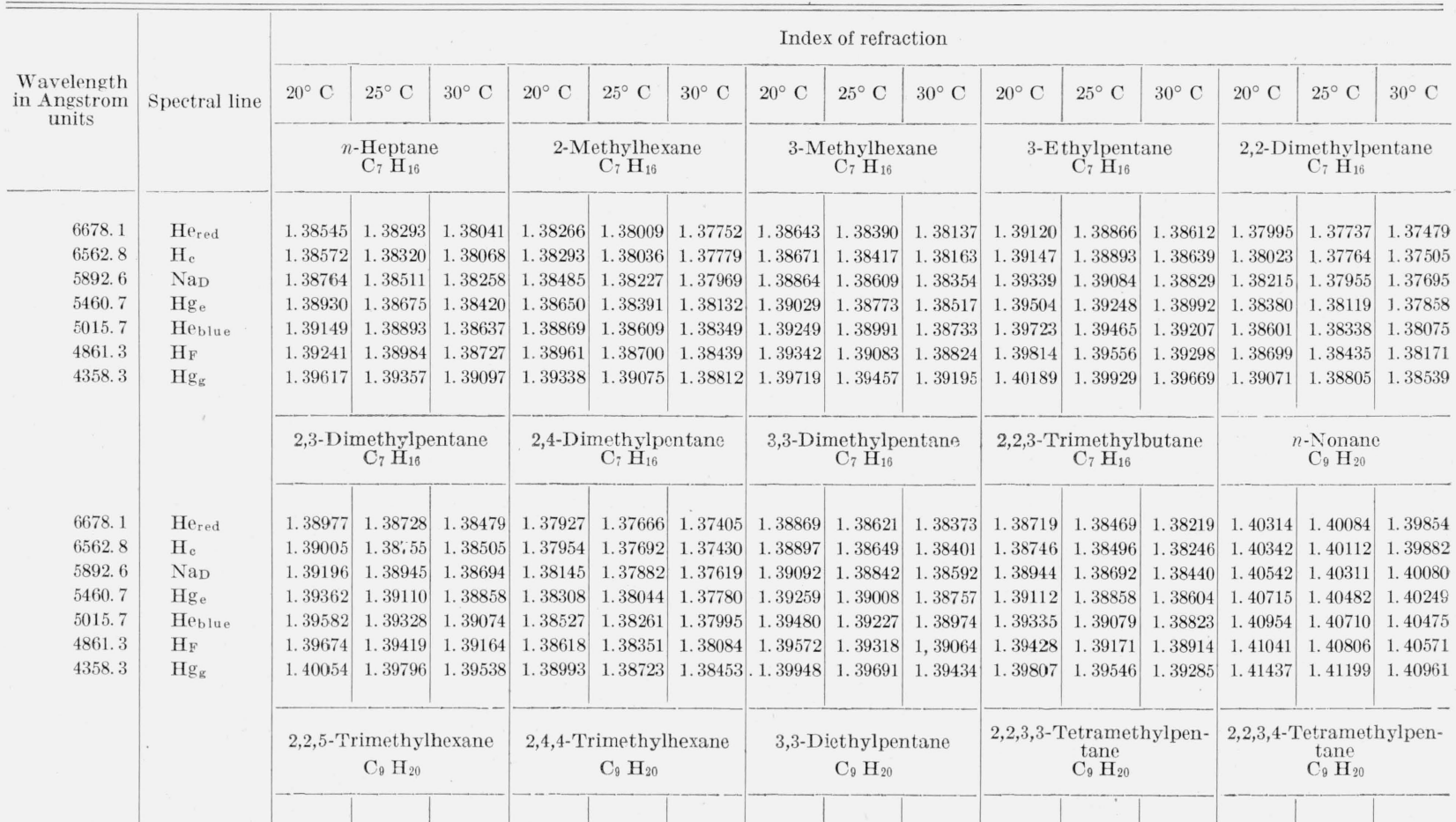

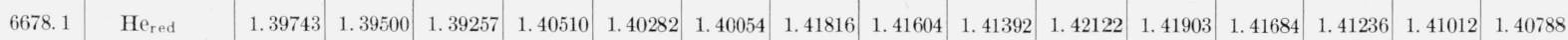
\begin{tabular}{|l|l|l|l|l|l|l|l|l|l|l|l|l|l|l|l|l|l|l|l}
6562.8 & $\mathrm{H}_{\mathrm{c}}$ & 1.39771 & 1.39528 & 1.39285 & 1.40540 & 1.40311 & 1.40082 & 1.41845 & 1.41633 & 1.41421 & 1.42152 & 1.41933 & 1.41714 & 1.41266 & 1.41041 & 1.40816 \\
\hline
\end{tabular}

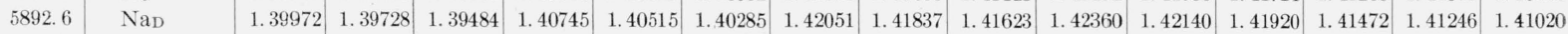

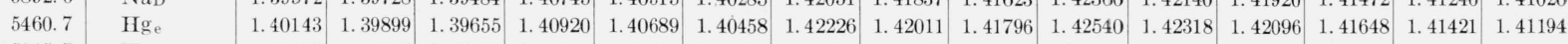

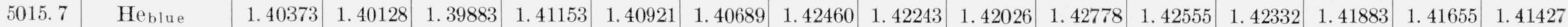
\begin{tabular}{l|l|l|l|l|l|l|l|l|l|l|l|l|l|l|l|l|l}
4861.3 & $\mathrm{H}_{\mathrm{F}}$ & 1.40470 & 1.40224 & 1.39978 & 1.41251 & 1.41018 & 1.40785 & 1.42558 & 1.42340 & 1.42122 & 1.42879 & 1.42655 & 1.42431 & 1.41981 & 1.41753 & 1.41525
\end{tabular}

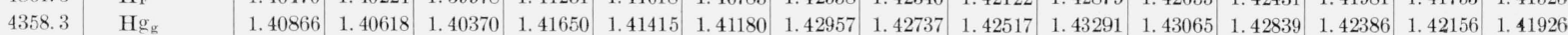

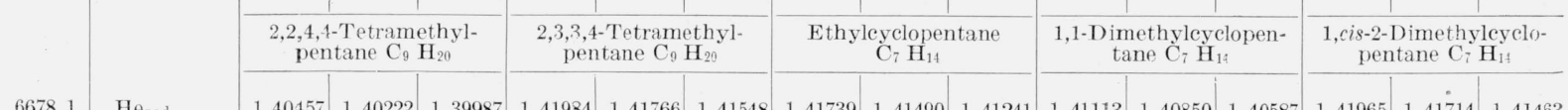

6er8.1 Hered

$6562.8 \quad \mathrm{H}_{\mathrm{c}}$

5892.6 Nap

$5460.7 \quad \mathrm{Hg}_{\mathrm{e}}$

5015.7 Heblue

4861.3 $\mathrm{H}_{\mathrm{F}}$

4358.3 $\quad \mathrm{Hg}_{\mathrm{g}}$

6678.1 Here

$6562.8 \mathrm{H}_{\mathrm{c}}$

5892.6 NaD

5460.7 $\mathrm{Hg}_{\mathrm{e}}$

5015.7 Heblue

4861. $3 \quad \mathrm{H}_{\mathrm{F}}$

4358. $3 \quad \mathrm{Hg}_{\underline{\underline{\sigma}}}$

6678. 1 He

$6562.8 \mathrm{H}_{\mathrm{c}}$

5892.6 NaD

5460.7 $\mathrm{Hg}_{\mathrm{e}}$

5015.7 Heblue

4861.3 $\quad \mathrm{H}_{\mathrm{F}}$

4358. $3 \quad \mathrm{Hg}_{\mathrm{g}}$ \begin{tabular}{|l|l|l|l|l|l|l|l|l|l|l|l|l|l|l}
1.40457 & 1.40222 & 1.39987 & 1.41984 & 1.41766 & 1.41548 & 1.41739 & 1.41490 & 1.41241 & 1.41113 & 1.40850 & 1.40587 & 1.41965 & 1.41714 & 1.41463
\end{tabular} \begin{tabular}{ll|l|l|l|l|l|l|l|l|l|l|l|l|l|l}
1.40487 & 1.40252 & 1.40017 & 1.42016 & 1.41798 & 1.41580 & 1.41769 & 1.41520 & 1.41271 & 1.41144 & 1.40880 & 1.40616 & 1.41997 & 1.41745 & 1.41493
\end{tabular} \begin{tabular}{l|l|l|l|l|l|l|l|l|l|l|l|l|l|l}
1.40694 & 1.40459 & 1.40224 & 1.42222 & 1.42003 & 1.41784 & 1.41981 & 1.41730 & 1.41479 & 1.41356 & 1.41091 & 1.40826 & 1.42217 & 1.41963 & 1.41709
\end{tabular} $\begin{array}{lllllllllllllllllll}1.40874 & 1.40638 & 1.40402 & 1.42402 & 1.42182 & 1.41962 & 1.42162 & 1.41910 & 1.41658 & 1.41538 & 1.41271 & 1.41004 & 1.42405 & 1.42148 & 1.41891\end{array}$ \begin{tabular}{l|l|l|l|l|l|l|l|l|l|l|l|l|l|l}
1.41111 & 1.40875 & 1.40639 & 1.42638 & 1.42417 & 1.42196 & 1.42401 & 1.42148 & 1.41895 & 1.41778 & 1.41510 & 1.41242 & 1.42649 & 1.42390 & 1.42131
\end{tabular} \begin{tabular}{ll|l|l|l|l|l|l|l|l|l|l|l|l|l|l}
1.41212 & 1.40975 & 1.40738 & 1.42737 & 1.42515 & 1.42293 & 1.42501 & 1.42247 & 1.41993 & 1.41878 & 1.41609 & 1.41340 & 1.42751 & 1.42490 & 1.42229
\end{tabular} \begin{tabular}{ll|l|l|l|l|l|l|l|l|l|l|l|l|l}
1.41623 & 1.41385 & 1.41147 & 1.43144 & 1.42920 & 1.42696 & 1.42910 & 1.42653 & 1.42396 & 1.42250 & 1.42018 & 1.41746 & 1.43161 & 1.42895 & 1.42629
\end{tabular}

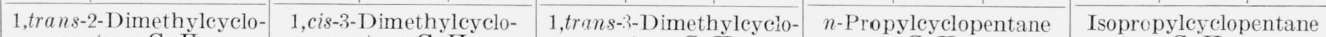

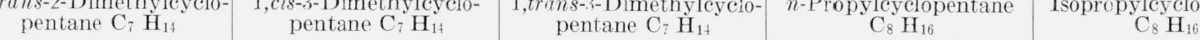

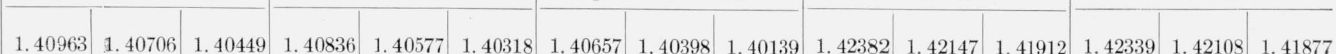

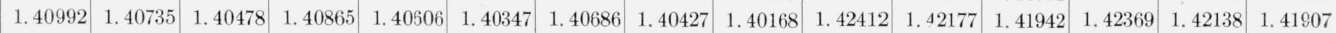

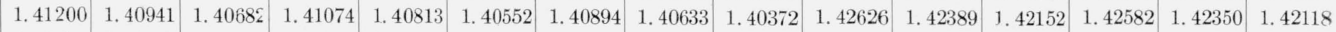
\begin{tabular}{|l|l|l|l|l|l|l|l|l|l|l|l|l|l|l|l}
1.41377 & 1.41117 & 1.40857 & 1.41250 & 1.40988 & 1.40726 & 1.41071 & 1.40809 & 1.40547 & 1.42809 & 1.42571 & 1.42333 & 1.42716 & 1.42532 & 1.42298
\end{tabular} \begin{tabular}{ll|l|l|l|l|l|l|l|l|l|l|l|l|l}
1.41612 & 1.41351 & 1.41080 & 1.41487 & 1.41223 & 1.40959 & 1.41307 & 1.41044 & 1.40781 & 1.43055 & 1.42814 & 1.42573 & 1.43010 & 1.42775 & 1.42540
\end{tabular} \begin{tabular}{ll|l|l|l|l|l|l|l|l|l|l|l|l|l|l}
1.41717 & 1.41455 & 1.41193 & 1.41586 & 1.41321 & 1.41056 & 1.41411 & 1.41147 & 1.40383 & 1.43156 & 1.42915 & 1.42674 & 1.43112 & 1.42876 & 1.42640
\end{tabular} \begin{tabular}{|l|l|l|l|l|l|l|l|l|l|l|l|l|l|l|l}
1.42118 & 1.41853 & 1.41588 & 1.41989 & 1.41721 & 1.41453 & 1.41812 & 1.41545 & 1.41278 & 1.43577 & 1.43332 & 1.43087 & 1.43533 & 1.43294 & 1.43055
\end{tabular}

\begin{tabular}{|c|c|c|c|c|}
\hline $\begin{array}{c}\text { 1-Methyl-1-ethylcyclo- } \\
\text { pentane } \mathrm{C}_{8} \mathrm{H}_{16}\end{array}$ & $\begin{array}{c}\text { 1-Methyl-cis-2-ethylcy- } \\
\text { clopentane } \mathrm{C}_{8} \mathrm{H}_{16}\end{array}$ & $\begin{array}{c}1,1,2 \text {-Trimethyleyclo- } \\
\text { pentane } \mathrm{C}_{8} \mathrm{H}_{16}\end{array}$ & $\begin{array}{c}1,1,3 \text {-Trimethylcyclo- } \\
\text { pentane } \mathrm{C}_{8} \mathrm{H}_{16}\end{array}$ & $\begin{array}{c}\text { Trimethylcyclopentane } \\
\mathrm{C}_{8} \mathrm{H}_{16}\end{array}$ \\
\hline
\end{tabular}

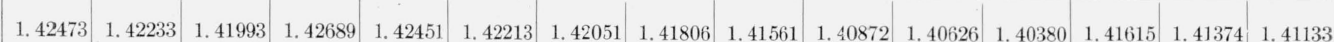
\begin{tabular}{ll|l|l|l|l|l|l|l|l|l|l|l|l|l|l}
1.42501 & 1.42261 & 1.42021 & 1.42719 & 1.42481 & 1.42243 & 1.42081 & 1.41836 & 1.41591 & 1.40804 & 1.40657 & 1.40410 & 1.41644 & 1.41403 & 1.41162
\end{tabular} \begin{tabular}{ll|l|l|l|l|l|l|l|l|l|l|l|l|l}
1.42718 & 1.42476 & 1.42234 & 1.42933 & 1.42695 & 1.42457 & 1.42298 & 1.42051 & 1.41804 & 1.41119 & 1.40870 & 1.40621 & 1.41855 & 1.41612 & 1.41369
\end{tabular} \begin{tabular}{|l|l|l|l|l|l|l|l|l|l|l|l|l|l|l}
1.42903 & 1.42660 & 1.42417 & 1.43116 & 1.42878 & 1.42640 & 1.42482 & 1.42234 & 1.41986 & 1.41302 & 1.41051 & 1.40799 & 1.42035 & 1.41791 & 1.41547
\end{tabular} \begin{tabular}{ll|l|l|l|l|l|l|l|l|l|l|l|l|l}
1.43148 & 1.42903 & 1.42657 & 1.43360 & 1.43121 & 1.42882 & 1.42728 & 1.42478 & 1.42228 & 1.41543 & 1.41290 & 1.41037 & 1.42276 & 1.42030 & 1.41784
\end{tabular} \begin{tabular}{ll|l|l|l|l|l|l|l|l|l|l|l|l|l}
1.43251 & 1.43005 & 1.42759 & 1.43461 & 1.43222 & 1.42983 & 1.42830 & 1.42580 & 1.42330 & 1.41644 & 1.41389 & 1.41134 & 1.42376 & 1.42129 & 1.41882
\end{tabular} \begin{tabular}{l|l|l|l|l|l|l|l|l|l|l|l|l|l|l|l|l}
1.43671 & 1.43421 & 1.43171 & 1.43877 & 1.43637 & 1.43397 & 1.43251 & 1.42998 & 1.42745 & 1.42053 & 1.41794 & 1.41535 & 1.42787 & 1.42537 & 1.42287
\end{tabular} 
TABLE 6.-Refractive indices at seven wavelengths and three temperatures for 60 API-NBS hydrocarbons--Continued

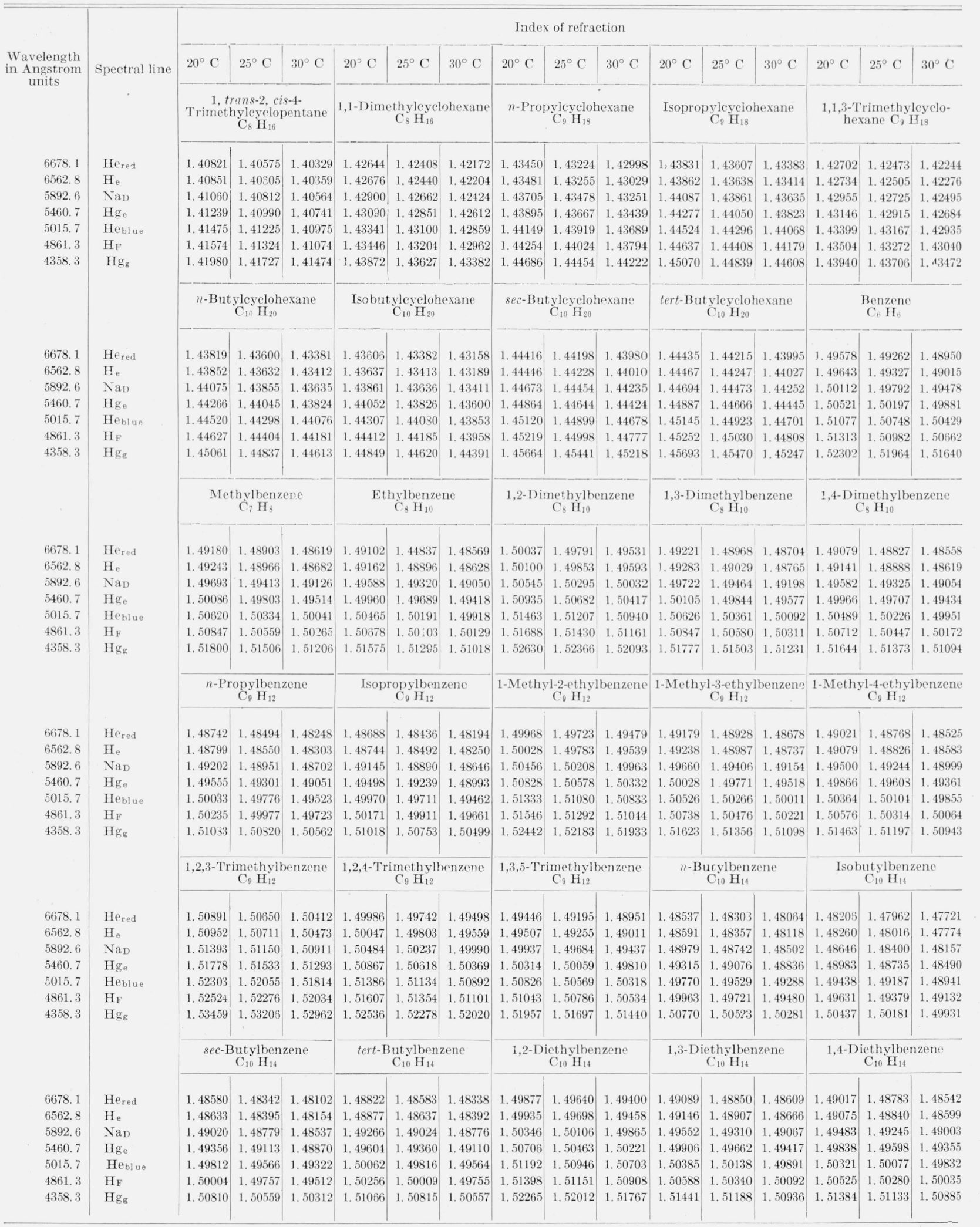


TABLE 7. Calculated values of the specific dispersion for 60 API-NBS hydrocarbons

\begin{tabular}{|c|c|c|c|c|c|c|c|c|c|c|}
\hline $\begin{array}{l}\text { Temper- } \\
\text { ature }\end{array}$ & $10^{4}\left(n_{F}-n_{C}\right) / d$ & $10^{4}\left(n_{g}-n_{D}\right) / d$ & $10^{4}\left(n_{F}-n_{C}\right) / d$ & $10^{4}\left(n_{g}-n_{D}\right) / d$ & $10^{4}\left(n_{F}-n_{C}\right) / d$ & $10^{4}\left(n_{g}-n_{D}\right) / d$ & $10^{4}\left(n_{F}-n_{C}\right) / d$ & $10^{4}\left(n_{g}-n_{D}\right) / d$ & $10^{4}\left(n_{F}-n_{C}\right) / d$ & $10^{4}\left(n_{g}-n_{D}\right) / d$ \\
\hline${ }^{\circ} \mathrm{C}$ & \multicolumn{2}{|c|}{$n$-Heptane } & \multicolumn{2}{|c|}{ 2-Methylhexane } & \multicolumn{2}{|c|}{ 3-Methylhexane } & \multicolumn{2}{|c|}{ 3-Ethylpentane } & \multicolumn{2}{|c|}{ 2,2-Dimethylpentane } \\
\hline $\begin{array}{l}20 \\
25 \\
30\end{array}$ & $\begin{array}{l}97.8 \\
97.7 \\
97.6\end{array}$ & $\begin{array}{l}124.8 \\
124.5 \\
124.2\end{array}$ & $\begin{array}{l}98.4 \\
98.5 \\
98.5\end{array}$ & $\begin{array}{l}125.7 \\
125.7 \\
125.8\end{array}$ & $\begin{array}{l}97.7 \\
97.5 \\
97.4\end{array}$ & $\begin{array}{l}124.4 \\
124.2 \\
123.9\end{array}$ & $\begin{array}{l}95.5 \\
95.5 \\
95.6\end{array}$ & $\begin{array}{l}121.7 \\
121.8 \\
121.8\end{array}$ & $\begin{array}{l}100.3 \\
100.2 \\
100.1\end{array}$ & $\begin{array}{l}127.0 \\
127.0 \\
126.9\end{array}$ \\
\hline 30 & \multicolumn{2}{|c|}{ 2,3-Dimethylpentane } & \multicolumn{2}{|c|}{ 2,4-Dimethylpentane } & \multicolumn{2}{|c|}{ 3,3-Dimethylpentane } & \multicolumn{2}{|c|}{ 2,2,3-Trimethylbutane } & \multicolumn{2}{|c|}{$n$-Nonane } \\
\hline $\begin{array}{l}20 \\
25 \\
30\end{array}$ & $\begin{array}{l}96.2 \\
96.1 \\
96.0\end{array}$ & $\begin{array}{l}123.4 \\
123.2 \\
122.9\end{array}$ & $\begin{array}{l}98.7 \\
98.6 \\
98.5\end{array}$ & $\begin{array}{l}126.0 \\
125.8 \\
125.6\end{array}$ & $\begin{array}{l}97.4 \\
97.1 \\
96.8\end{array}$ & $\begin{array}{l}123.5 \\
123.2 \\
123.0\end{array}$ & $\begin{array}{l}98.8 \\
98.4 \\
98.0\end{array}$ & $\begin{array}{l}125.0 \\
124.5 \\
124.0\end{array}$ & $\begin{array}{l}97.4 \\
97.2 \\
97.0\end{array}$ & $\begin{array}{l}124.7 \\
124.4 \\
124.1\end{array}$ \\
\hline 30 & \multicolumn{2}{|c|}{ 2,2,5-Trimethylhexane } & \multicolumn{2}{|c|}{ 2,4,4-Trimethylhexane } & \multicolumn{2}{|c|}{ 3,3-Diethylpentane } & \multicolumn{2}{|c|}{$\begin{array}{l}\text { 2,2,3,3-Tetramethyl- } \\
\text { pentane }\end{array}$} & \multicolumn{2}{|c|}{$\begin{array}{l}\text { 2,2,3,4-Tetramethyl- } \\
\text { pentane }\end{array}$} \\
\hline $\begin{array}{l}20 \\
25 \\
30\end{array}$ & $\begin{array}{l}98.8 \\
99.0 \\
99.1\end{array}$ & $\begin{array}{l}126.4 \\
126.6 \\
126.7\end{array}$ & $\begin{array}{l}98.2 \\
98.2 \\
98.2\end{array}$ & $\begin{array}{l}125.0 \\
125.0 \\
125.0\end{array}$ & $\begin{array}{l}94.6 \\
94.3 \\
93.9\end{array}$ & $\begin{array}{l}120.2 \\
120.0 \\
119.8\end{array}$ & $\begin{array}{l}96.1 \\
95.9 \\
95.7\end{array}$ & $\begin{array}{l}123.0 \\
122.8 \\
122.6\end{array}$ & $\begin{array}{l}96.8 \\
96.8 \\
96.9\end{array}$ & $\begin{array}{l}123.7 \\
123.8 \\
123.9\end{array}$ \\
\hline 30 & \multicolumn{2}{|c|}{$\begin{array}{l}\text { 2,2,4,4-Tetramethyl- } \\
\text { pentane }\end{array}$} & \multicolumn{2}{|c|}{$\begin{array}{l}\text { 2,3,3,4-Tetramethyl- } \\
\text { pentane }\end{array}$} & \multicolumn{2}{|c|}{ Ethylcyclopentane } & \multicolumn{2}{|c|}{$\begin{array}{l}\text { 1,1-Dimethylcyclo- } \\
\text { pentane }\end{array}$} & \multicolumn{2}{|c|}{$\begin{array}{l}\text { 1,cis-2-Dimethylcyclo- } \\
\text { pentane }\end{array}$} \\
\hline $\begin{array}{l}20 \\
25 \\
30\end{array}$ & $\begin{array}{l}100.8 \\
101.0 \\
101.3\end{array}$ & $\begin{array}{l}129.1 \\
129.4 \\
129.7\end{array}$ & $\begin{array}{l}95.5 \\
95.4 \\
95.4\end{array}$ & $\begin{array}{l}122.2 \\
122.1 \\
122.0\end{array}$ & $\begin{array}{l}95.5 \\
95.4 \\
95.3\end{array}$ & $\begin{array}{l}121.2 \\
121.1 \\
121.0\end{array}$ & $\begin{array}{l}97.3 \\
97.2 \\
97.1\end{array}$ & $\begin{array}{l}123.8 \\
123.6 \\
123.4\end{array}$ & $\begin{array}{l}97.6 \\
97.0 \\
96.4\end{array}$ & $\begin{array}{l}122.2 \\
121.3 \\
120.4\end{array}$ \\
\hline 30 & \multicolumn{2}{|c|}{$\begin{array}{l}\text { 1,trans-2-Dimethylcy- } \\
\text { clopentane }\end{array}$} & \multicolumn{2}{|c|}{$\begin{array}{l}\text { 1,cis-3-Dimethylcyclo- } \\
\text { pentane }\end{array}$} & \multicolumn{2}{|c|}{$\begin{array}{l}\text { 1,trans-3-Dimethylcy- } \\
\text { clopentane }\end{array}$} & \multicolumn{2}{|c|}{$n$-Propylcyclopentane } & \multicolumn{2}{|c|}{ Isopropylcyclopentane } \\
\hline \multirow[t]{2}{*}{30} & $\begin{array}{l}96.5 \\
96.4 \\
96.3\end{array}$ & $\begin{array}{l}122.2 \\
122.1 \\
122.0\end{array}$ & $\begin{array}{l}96.3 \\
96.0 \\
95.8\end{array}$ & $\begin{array}{l}122.2 \\
122.0 \\
121.8\end{array}$ & $\begin{array}{l}97.3 \\
97.3 \\
97.2\end{array}$ & $\begin{array}{l}123.2 \\
123.2 \\
123.1\end{array}$ & $\begin{array}{l}95.8 \\
95.6 \\
95.3\end{array}$ & $\begin{array}{l}122.5 \\
122.1 \\
121.7\end{array}$ & $\begin{array}{l}95.7 \\
95.5 \\
95.4\end{array}$ & $\begin{array}{l}122.5 \\
122.2 \\
121.9\end{array}$ \\
\hline & \multicolumn{2}{|c|}{$\begin{array}{l}\text { 1-Methyl-1-ethylcyclo- } \\
\text { pentane }\end{array}$} & \multicolumn{2}{|c|}{$\begin{array}{l}\text { 1-Methyl-cis-2-ethylcyclo- } \\
\text { pentane }\end{array}$} & \multicolumn{2}{|c|}{$\begin{array}{l}\text { 1,1,2-Trimethylcyclo- } \\
\text { pentane }\end{array}$} & $\begin{array}{l}\text { 1,1,3-Trime } \\
\text { pent }\end{array}$ & $\begin{array}{l}\text { thylcyclo- } \\
\text { ane }\end{array}$ & $\begin{array}{r}1, \text { cis-2,trans-4 } \\
\text { cyclope }\end{array}$ & $\begin{array}{l}\text {-Trimethyl- } \\
\text { entane }\end{array}$ \\
\hline $\begin{array}{l}20 \\
25 \\
30\end{array}$ & $\begin{array}{l}96.0 \\
95.8 \\
95.5\end{array}$ & $\begin{array}{l}122.0 \\
121.7 \\
121.3\end{array}$ & $\begin{array}{l}94.5 \\
94.9 \\
95.2\end{array}$ & $\begin{array}{l}120.2 \\
120.6 \\
121.0\end{array}$ & $\begin{array}{l}97.0 \\
96.9 \\
96.7\end{array}$ & $\begin{array}{l}123.4 \\
123.3 \\
123.2\end{array}$ & $\begin{array}{l}98.9 \\
98.4 \\
97.9\end{array}$ & $\begin{array}{l}124.8 \\
124.2 \\
123.6\end{array}$ & $\begin{array}{l}95.9 \\
95.6 \\
95.4\end{array}$ & $\begin{array}{l}122.1 \\
121.8 \\
121.6\end{array}$ \\
\hline & $\begin{array}{r}\text { 1,trans-2,cis-4 } \\
\text { cyclope }\end{array}$ & $\begin{array}{l}\text { 4-Trimethyl- } \\
\text { entane }\end{array}$ & 1,1-Dimethyl & leyclohexane & $n$-Propylcy & clohexane & Isopropyley & yclohexane & $\begin{array}{r}1,1,3-\text { Trim } \\
\text { hexa }\end{array}$ & $\begin{array}{l}\text { lethylcyclo- } \\
\text { ane }\end{array}$ \\
\hline $\begin{array}{l}20 \\
25 \\
30\end{array}$ & $\begin{array}{l}96.8 \\
96.8 \\
96.8\end{array}$ & $\begin{array}{l}123.1 \\
123.1 \\
123.2\end{array}$ & $\begin{array}{l}98.6 \\
98.4 \\
98.1\end{array}$ & $\begin{array}{l}124.5 \\
124.2 \\
124.0\end{array}$ & $\begin{array}{l}97.4 \\
97.4 \\
97.4\end{array}$ & $\begin{array}{l}123.6 \\
123.6 \\
123.6\end{array}$ & $\begin{array}{l}96.6 \\
96.5 \\
96.3\end{array}$ & $\begin{array}{l}122.5 \\
122.5 \\
122.5\end{array}$ & $\begin{array}{l}98.9 \\
99.0 \\
99.1\end{array}$ & $\begin{array}{l}126.5 \\
126.6 \\
126.7\end{array}$ \\
\hline & $n$-Butylcy & clohexane & Isobutylcy & clohexane & sec-Butylcy & yclohexane & tert-Butyle. & yclohexane & Ber & nzene \\
\hline $\begin{array}{l}20 \\
25 \\
30\end{array}$ & $\begin{array}{l}97.0 \\
97.0 \\
97.1\end{array}$ & $\begin{array}{l}123.4 \\
123.4 \\
123.5\end{array}$ & $\begin{array}{l}97.4 \\
97.5 \\
97.6\end{array}$ & $\begin{array}{l}124.2 \\
124.3 \\
124.4\end{array}$ & $\begin{array}{l}95.1 \\
95.1 \\
95.2\end{array}$ & $\begin{array}{l}121.9 \\
121.9 \\
122.0\end{array}$ & $\begin{array}{l}96.6 \\
96.8 \\
97.0\end{array}$ & $\begin{array}{l}122.9 \\
123.2 \\
123.6\end{array}$ & $\begin{array}{l}190.0 \\
189.4 \\
189.6\end{array}$ & $\begin{array}{l}249.1 \\
248.6 \\
248.9\end{array}$ \\
\hline & Methylk & benzene & Ethylb & enzene & 1,2-Dimeth & ylbenzene & 1,3-Dimeth & ylbenzene & 1,4-Dimeth & ylbenzene \\
\hline 20 & 185.0 & 243.0 & 174.9 & 229.2 & 180.4 & 236.9 & 181.0 & 237.8 & 182.4 & 239.5 \\
\hline 25 & 184.7 & 242.7 & 174. 7 & 228.9 & 180.0 & 236.4 & 180.4 & 237.1 & 182.0 & 239.0 \\
\hline 30 & 184.6 & 242.5 & 174.9 & 229.3 & 179.8 & 236. 3 & 180.7 & 237.6 & 182.2 & 239.3 \\
\hline
\end{tabular}


TABLE 7. Calculated values of the specific dispersion for 60 API-NBS hydrocarbons-Continued

\begin{tabular}{|c|c|c|c|c|c|c|c|c|c|c|}
\hline $\begin{array}{l}\text { Temper- } \\
\text { ature }\end{array}$ & $10^{4}\left(n_{F}-n_{C}\right) / d$ & $10^{4}\left(n_{g}-n_{D}\right) / d$ & $10^{4}\left(n_{F}-n_{C}\right) / d$ & $10^{4}\left(n_{g}-n_{D}\right) / d$ & $10^{4}\left(n_{F}-n_{C}\right) / d$ & $10^{4}\left(n_{g}-n_{D}\right) / d$ & $10^{4}\left(n_{F}-n_{C}\right) / d$ & $10^{4}\left(n_{g}-n_{D}\right) / d$ & $10^{4}\left(n_{F}-n_{C}\right) / d$ & $10^{4}\left(n_{g}-n_{D}\right) / d$ \\
\hline${ }^{\circ} \mathrm{C}$ & \multicolumn{2}{|c|}{$n$-Propylbenzene } & \multicolumn{2}{|c|}{ Isopropylbenzene } & \multicolumn{2}{|c|}{ 1-Methyl-2-ethylbenzene } & \multicolumn{2}{|c|}{ 1-Methyl-3-ethylbenzene } & \multicolumn{2}{|c|}{ 1-Methyl-4-ethylbenzene } \\
\hline 20 & 166.6 & 218.2 & 165.6 & 217.3 & 172.4 & 225.5 & 173.5 & 227.1 & 173.8 & 227.9 \\
\hline 25 & 166.4 & 217.9 & 165.5 & 217.2 & 172.1 & 225.3 & 173.0 & 226.6 & 173.6 & 227.9 \\
\hline \multirow[t]{2}{*}{30} & 166.3 & 217.9 & 165.3 & 217.1 & 172.5 & 225.8 & 173.3 & 227.0 & 173.6 & 227.9 \\
\hline & \multicolumn{2}{|c|}{ 1,2,3-Trimethylbenzene } & \multicolumn{2}{|c|}{ 1,2,4-Trimethylbenzene } & \multicolumn{2}{|c|}{ 1,3,5-Trimethylbenzene } & \multicolumn{2}{|c|}{$n$-Butylbenzene } & \multicolumn{2}{|c|}{ Isobutylbenzene } \\
\hline 20 & 175.8 & 231.0 & 378. 1 & 234.3 & 177.5 & 233.5 & 159.5 & 208. 2 & 160.7 & 209.9 \\
\hline 25 & 175.8 & 230.9 & 177.9 & 234.1 & 177.8 & 233.8 & 159.3 & 208.0 & 160.5 & 210.0 \\
\hline \multirow[t]{2}{*}{30} & 176.1 & 231.4 & 177.7 & 233.9 & 177.7 & 233.7 & 159.8 & 208.8 & 160.7 & 209.9 \\
\hline & \multicolumn{2}{|c|}{ sec-Butylbenzene } & \multicolumn{2}{|c|}{ tert-Butylbenzene } & \multicolumn{2}{|c|}{ 1,2-Diethylbenzene } & \multicolumn{2}{|c|}{ 1,3-Diethylbenzene } & \multicolumn{2}{|c|}{ 1,4-Diethylbenzene } \\
\hline 20 & 159.0 & 207.6 & 159.1 & 207.7 & 166. 2 & 218.1 & 166. 9 & 218.6 & 168. 2 & 220.5 \\
\hline 25 & 158.7 & 207.5 & 159.1 & 207.7 & 165.9 & 217.6 & 166. 6 & 218.4 & 167.8 & 220.1 \\
\hline 30 & 159.0 & 207.8 & 158.8 & 207.5 & 166.3 & 218.1 & 166.6 & 218.4 & 168.1 & 220.4 \\
\hline
\end{tabular}

\section{Discussion}

Figure 3 gives, for the several normal alkylbenzenes measured, plots of the values of the constants $n_{\infty}$ and $C$ in relation to the number of carbon atoms in the normal alkyl radical. It appears that these relations are such that, when one sufficiently higher member of the series, such as $n$-decylbenzene, is evaluated, it will be possible to set up the complete equation for the intermediate members of the series as well as for some members higher than $n$-decylbenzene, with a knowledge only of the refractive index at one wavelength, as $n_{D}$.

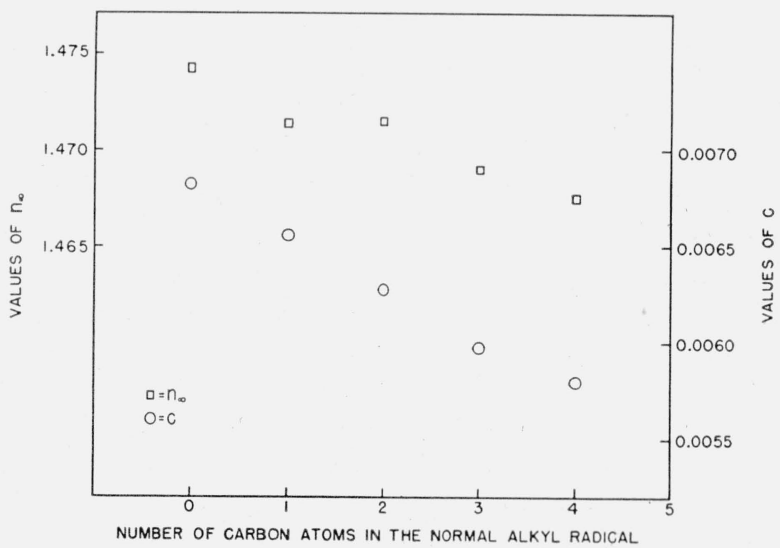

Figure 3 . Values of the constants, $n_{\infty}$ and $C$, of the Hartman equation, as a function of the number of carbon atoms in the normal alkyl radical of five alkylbenzenes.
It is planned to do this for the series of normal alkylbenzenes, normal monoolefins (l-alkenes), normal paraffins, normal alkylcyclopentanes, and normal alkylcyclohexanes.

Grateful acknowledgement is made to Frederick D. Rossini for his many suggestions in the course of the investigation and in the preparation of this report.

\section{References}

[1] G. B. Arnold and L. Donn, Anal. Chem. 19, 666 (1947).

[2] A. F. Forziati and F. D. Rossini, J. Research NBS 43, 473 (1949) RP 2038.

[3] A. R. Glasgow, Jr., E. T. Murphy, C. B. Willingham, and F. D. Rossini, J. Research NBS 37, 141 (1946) RP1734.

[4] A. J. Streiff, E. T. Murphy, V. A. Sedlak, C. B. Willingham, and F. D. Rossini, J. Research NBS 37, 331 (1946) RP1752.

[5] A. J. Streiff, E. T. Murphy, J. C. Cahill, H. F. Flanagan, V. A. Sedlak, C. B. Willingham, and F. D. Rossini, J. Research NBS 38, 53 (1947) RP1760.

[6] A. J. Streiff, E. T. Murphy, J. C. Zimmerman, L. F. Soule, V. A. Sedlak, C. B. Willingham, and F. D. Rossini, J. Research NBS 39, 321 (1947) RP1833.

[7] A. J. Streiff, J. C. Zimmerman, L. F. Soule, M. T. Butt, V. A. Sedlak, C. B. Willingham, and F. D. Rossini, J. Research NBS 41, 323 (1948) RP1929.

[8] L. W. Tilton and H. L. Gurewitz, NBS, private communication.

Washington, November 4, 1949. 